\title{
Effects of natural growth regulators on micropropagation of potatoes
}

\author{
Zaib-Un-Nisa ${ }^{*}$, Azhar Hussain Shah ${ }^{2}$, Safdar Hussain Shah ${ }^{3}$, Ghulam \\ Farooq $^{4}$, Muhammad Anwar Sajad ${ }^{5}$ and Muhammad Anwar Saleem \\ $\mathrm{Khan}^{6}$
}

1. Department of Botany, Women University Swabi, Khyber Pakhtunkhwa-Pakistan

2. Department of Botany, Hazara University, Mansehra, Khyber Pakhtunkhwa-Pakistan

3. Institute of Biotechnology and Genetic Engineering, Agriculture University, Peshawar, Khyber PakhtunkhwaPakistan

4. Department of Genetics, Hazara University, Mansehra, Khyber Pakhtunkhwa-Pakistan

5. Department of Botany, Islamia College (A Public Sector University) Peshawar, Peshawar, Khyber

Pakhtunkhwa-Pakistan

6. Department of Zoology, University of Swabi, Khyber Pakhtunkhwa-Pakistan

*Corresponding author's email: zaib2864@gmail.com

Citation

Zaib-Un-Nisa, Azhar Hussain Shah, Safdar Hussain Shah, Ghulam Farooq, Muhammad Anwar Sajad and Muhammad Anwar Saleem Khan. Effects of natural growth regulators on micropropagation of potatoes. Pure and Applied Biology. Vol. 9, Issue 2, pp1442-1459. http://dx.doi.org/10.19045/bspab.2020.90150

\begin{tabular}{|c|c|c|c|}
\hline Received: 19/12/2019 & Revised: $25 / 02 / 2020$ & Accepted: 06/03/2020 & Online First: 09/03/2020 \\
\hline
\end{tabular}

\section{Abstract}

To find the cheapest substitutes for synthetic growth regulators such as Gibberillic acid (GA3), natural growth substances that are not patent yet so named as TANG and ARQ, were evaluated for their performance in the Murashiage and Skoog (MS) medium for micropropagation of potatoes (Solanum tuberosum L.). Natural growth substances as synthetic growth regulators were used for their excellence performance in M.S media. Natural growth substances were the extract of plants and cheaper sources of plant growth regulators. In present study axillary shoots of potatoes plants were used as explants. After pilot study $14 \mathrm{ml} / \mathrm{l} \mathrm{TAN} \mathrm{G}$ and $10 \mathrm{ml} / \mathrm{l} \mathrm{ARQ}$ were selected as suitable concentrations to be used for experiments. Data were recorded for 3 , 6, 8, 10 and 15 days. Overall growth response of TANG and ARQ supplemented medium was very similar rather to standard medium having GA3, with no adverse effect on plants health. This MS medium would approximately reduce the production to one half of the conventional methodology. This strongly suggests that natural growth substances (TANG and ARQ) as growth stimulators are the most suitable and a cheaper alternative for very expensive and more frequently used constitutes of MS medium for micropropagation of potatoes.

Keywords: Micropropagation; Natural growth regulators; Solanum tuberosum L.; Synthetic growth regulator

\section{Introduction}

Potato (Solanum tuberosum L.) is an important food crop belongs to the family Solanaceae. It is a native to the Andean highlands of South America, where, it has served as staple food for centuries or millennia. In recent years potato has been cultivated in many countries of the world with warmer and drier climates. Potato growers produce $325 \times 10^{6}$ thousand ton potatoes annually all over the world [1]. It is good and cheap source of carbohydrates, vitamins, minerals, and proteins. It also provides most of the trace elements which can meet the energy and dietary requirements of people living in the developing countries like Pakistan [2]. Potato has a wide distribution over the 
globe and grown successfully in dry climates like Pakistan. It was grown on an area of 127.7 thousand hectares with total annual production of 3726.5 thousand tons during 2010-2011 [3]. However, the present National yield is far lower than the production in developed countries. Main causes of low production are poor agronomic practices and non-availability of certified seeds (quality disease free seed). Besides these factors maintenance of potato germplasm through field clonal propagation is time-consuming and requires large amounts of space and is laborious. This also exposes the plants to diseases, pests, and risks of yield loss due to abiotic stresses and natural calamities [4].

On the other hand, the total seed provided by the public was 132.2 tons and 4283.2 tons were imported; total seed availability was 4,415.4 tons, which was lower than the national demand [3]. To narrow the gap between seed demand and supply, to minimize the foreign exchange spending on seed import, to reduce propagation time, labor cost and risks of diseases the sustainable strategy would be to accelerate and strengthen seed production programs [4]. As potatoes are normally vegetatively propagated, contaminations of seed material by pathogens (bacteria, virus and fungi) cause severe reduction in yield [5]. About twenty viruses are known to infect potato. While most important are Potex virus $\mathrm{X}$ (PVX) and Potex virus Y (PVY) which cause severe damage to potato crop in Pakistan [6]. Potex virus $\mathrm{X}$ (PVX) is a latent virus, therefore, sometimes unable to identify on symptom basis.

Clonal propagation is desirable for large scale or commercial production because it has an advantage of immediate 'age transfer' that saves time, reduce cost and the only way to conserve true to type plant $[7,8]$. But lack of budget, limited resource allocation and relatively high recurrent cost (chemical expenses) of this technology has been envisaged as a major obstacle in benefiting from this technology especially for developing countries like in Pakistan. The only alternative is to develop costeffective technology for in vitro clonal propagation of disease free potato that requires controlled environment and defined medium. The principal components of most plant tissue culture media are inorganic nutrients (macronutrients and micronutrients) carbon source (sucrose) organic supplements (vitamins), gelling agent (agar) and growth regulators. The most expensive components of media are growth regulators which are most frequently used for root and shoot initiation in tissue culture media [9]. Therefore, in the present research work an attempt has been made to substitute synthetic growth regulators by natural growth substances to decrease the cost per plantlet with the objectives to select a cheaper substitute for synthetic regulators. Secondly, to test the efficacy of different natural growth substances in comparison with synthetic regulators for micro propagation of potato. For this purpose different parameters were studied which were: Days to root initiation, Number of roots plant ${ }^{-1}$, Days to bud proliferation, Shoot length plant $^{-1}$ and Number of nodes plant ${ }^{-1}$.

\section{Materials and methods}

\section{Media for micropropagation}

The procedure followed by Nisa et al. [1014] was used for micropropagation. The basal Murashige and Skoog medium with minor modification was used for micropropagation.

Growth Regulators, $10 \mathrm{mg}$ Gibberillic acid (GA3) was dissolved in $50 \mathrm{ml}$ distilled water, made the volume up to $100 \mathrm{ml}$ and stored in a refrigerator at $4{ }^{\circ} \mathrm{C}$ [11].

\section{Sterilization of Media}

Media were dispensed in aliquots of 15 $\mathrm{ml} /$ tube $(15 \times 30 \mathrm{~mm})$; test tubes and other equipment (scalpel, Pasture pipettes and forceps) were sterilized by autoclaving at p.s.i. at $121{ }^{\circ} \mathrm{C}$ for 20 minutes [10-14].

\section{Explants source and Preparation of} plant materials

Explants were taken from the green house of Institute of Biotechnology and Genetic 
Engineering (IBGE) Khyber Pakhtunkhwa (KP) Agricultural University, Peshawar. Potato was used for single nodal cutting and its further propagation. In tissue culture technology explants physiological condition is crucial for morphogenesis. To maintain the physiological uniformity of explants three apical nodes were used throughout the experiment [11].

\section{Preparation of explants and culturing}

The dissecting operation was performed in the laminar flow. Before dissecting the explants, hands were washed with $75 \%$ ethanol, all the glassware was washed with detergent. Rinsed several times with distilled water and dried. Dissecting equipments e.g. blades, forceps and scalpel were also sterilized for aseptic manipulation. To minimize the chance of infection instruments were dipped in disinfectant and flamed after each operation.

The leaves were stripped away and single node cuttings (SNCs) were dissected from aseptically grown plants in test tubes and cultured/inoculated in vertical position in test tubes containing $15 \mathrm{ml} \mathrm{MS}$ medium supplemented with natural growth regulators and synthetic growth regulator $[11,12]$.

\section{Measurements of growth}

Growth of the shoots was measured by the methods of Martinez et al. [15] i.e. shoot length $(\mathrm{cm})$ after 3, 6, 8, 10, 13 and 15 days of culturing.

There was no consistency in root length, therefore; no root measurement was subsequently used.

\section{Acclimatization and growth in green house}

After 15 days of culturing plantlets were transferred from test tubes to small pots containing soil for hardening and acclimatization and were kept under sterile conditions for few days and were subsequently transferred to the green house [11]. Statistical analysis

"Analysis Tool Pak" of excel was used for analysis of variance and graphs.

\section{Results and discussion}

Much work has been done on plant extracts which were used for the management of diseases /pests. Terlizzi et al. [16] worked on extracts of barley straw to recognize the effects on alga flagella. Raguraman and Singh [17] worked on ethanol extract of neem seed kernel to evaluate the insects' effect. Plant extracts (Onion, Ginger and Garlic) were effective in reducing the disease frequency in very low concentrations i.e. at $1 \%$. Garlic extract at $1 \%$ concentration showed superior results as compared to control and other plant extracts in controlling the disease frequency against PVY but in case of PVX onion indicated healthier results as compared to control and other plant extracts. It might be accomplished that plant extracts play very important role in the management of viral diseases [18].

In plant tissues some chemicals naturally occurring and these chemicals have a regulatory role, instead of the nutritional role in growth and development. These chemical compounds are generally active at very low concentrations and are called plant hormones or plant growth substances. Synthetic growth regulators with similar physiological activities to plant growth substances, having an ability to alter plant growth. Similar results were also proposed by Nisa et al. $[12,14]$. Some of the natural growth substances are prepared synthetically [19].

Potato is usually vegetatively propagated. A drastic reduction in its production is caused by viral and fungal diseases and also by low quality and uncertified seeds. To overcome these problems in vitro micropropagation is the only solution. Tissue culture is the most effective and well known technique through which disease free plantlets are produced in a very short time and in large number [10-15]. But in tissue culture the high cost of synthetic growth regulators limits its application especially in developing countries. For this purpose natural growth regulatory extracts, TANG and ARQ were used as a substitute 
of synthetic growth regulators, prepared at the Institute of Biotechnology and Genetic Engineering (IBGE), Peshawar.

In the present study, apical or auxiliary shoots were used as explants for the purpose of in vitro micro propagation as reported by Nisa et al. [11] and Mohammadi and Sivritepe [20] that propagation from nodes and shoot tip explants are frequently preferred for commercial mass propagation purposes. The explants were properly sterilized and then cultured under aseptic conditions on Murashiage and Skoog, media supplemented with natural growth regulators and synthetic growth regulator separately.

Root initiation started on second day of culturing in synthetic as well as in natural growth substances. Maximum numbers of roots were observed in the medium supplemented with ARQ (1.87) whereas the medium containing GA3 (1.75) was second to it while minimum numbers of roots (1.375) were observed in medium having TANG (Fig. 1). Statistical analysis shows that there was no significant difference between the natural growth substances and synthetic growth regulators.

Maximum numbers of roots (2.12) were observed in the medium supplemented with ARQ. The medium numbers of roots (1.75) were observed in medium containing GA3 while the medium having TANG gave minimum number (1.375) of roots after three days of culturing (Fig. 2). Statistical analysis shows that there was no significant difference between the natural growth substances and synthetic growth regulators. Data recorded after six days of culturing indicated that medium containing ARQ induced maximum number of roots (7.37) and TANG containing medium induced medium number (6.37) of roots and was second to it. Whereas the medium supplemented with GA3 showed minimum number (2.87) of roots plant ${ }^{-1}$ (Fig. 3). Statistical analysis shows that there was no significant difference between the natural growth substances and synthetic growth regulators.

After eight days of culturing the recorded data showed that the medium supplemented with ARQ induced maximum (10) number of roots while TANG contained medium induced (7.62) number of roots and second to it. GA3 containing medium showed minimum number (4) of roots plant ${ }^{-1}$ (Fig. 4). Statistical analysis shows that there was no significant difference between the natural growth substances and synthetic growth regulators.

The data recorded after ten days of culturing indicated that the medium supplemented with ARQ induced maximum (13.75) number of roots and TANG containing medium induced medium number (11) of roots and was second to it. Whereas the medium having GA3 showed minimum (5.75) number of roots plant $^{-1}$ (Fig. 5). Statistical analysis shows that there was no significant difference between the natural growth substances and synthetic growth regulators. The data recorded after thirteen days of culturing indicated that ARQ containing medium induced maximum (17.5) number of roots while the medium having TANG (13.25) was second to it. The medium supplemented with GA3 showed minimum (6.8) number of roots plant ${ }^{-1}$ (Fig. 6). Statistical analysis shows that there was significant difference between the natural growth substances and synthetic growth regulators.

The data recorded after fifteen days of culturing indicated that the medium having ARQ induced maximum (18.87) number of roots whereas the medium supplemented with TANG (13.37) was second to it. GA3 containing medium showed minimum (6.87) number of roots plant ${ }^{-1}$ (Fig. 7). Statistical analysis shows that there was significant difference between the natural growth substances and synthetic growth regulators.

Bud initiation mostly started on second day of culturing in synthetic as well as in natural growth substances. It can be seen from (Fig. 
8) that over all response of all the three growth substances were parallel to bud proliferation i.e. almost all bud proliferation completed up to third day of inoculation. Statistical analysis shows that there was no significant difference between the natural growth substances and synthetic growth regulators. These results are in agreement with Shah et al. [21] and Nisa et al. [11]. Who also reported that both (natural and synthetic) growth regulators induced bud proliferation after the same number of days.

The average number of shoots plant ${ }^{-1}$ after six days of culturing indicated that the medium containing TANG produced maximum (1.37) number of shoots were observed whereas the medium supplemented with GA3 (1.12) and the medium having ARQ (1.12) induced similar number of shoots plant ${ }^{-1}$ (Fig. 9). Statistical analysis shows that there was no significant difference between the natural growth substances and synthetic growth regulators.

The average number of shoots plant ${ }^{-1}$ after eight days of culturing indicated that maximum (1.75) number of shoots observed in the medium containing TANG whereas, the medium having GA3 (1.62) was second to it while ARQ supplemented medium induced minimum (1.37) number of shoots plant ${ }^{-1}$ (Fig. 10). Statistical analysis shows that there was no significant difference between the natural growth substances and synthetic growth regulators. The average number of shoots plant ${ }^{-1}$ after ten days of culturing indicated that the medium containing TANG produced maximum (2) number of shoots whereas synthetic growth regulator, the medium supplemented with GA3 (1.62) was second to it and the medium containing ARQ was induced minimum (1.5) number of shoots plant $^{-1}$ (Fig. 11). Statistical analysis shows that there was no significant difference between the natural growth substances and synthetic growth regulators.

The average number of shoots plant ${ }^{-1}$ after thirteen days of culturing indicated that the medium having TANG (2.625) and the medium supplemented with ARQ (2.62) induced similar number of shoots plant ${ }^{-1}$ whereas the medium containing GA3 (2) was second to them (Fig. 12). Statistical analysis shows that there was no significant difference between the natural growth substances and synthetic growth regulator. The average number of shoots plant ${ }^{-1}$ after fifteen days of culturing indicated that the medium containing TANG produced maximum (3.5) number of shoots and the medium supplemented with ARQ (2.37) was second to it whereas the medium containing GA3 was induced minimum (2.12) number of shoots plant ${ }^{-1}$ (Fig. 13). Statistical analysis shows that there was no significant difference between the natural growth substances and synthetic growth regulator. The natural growth regulators when used in specific concentration stimulate root growth and development and also enhance shoot growth. These results are supported by Wang et al. [22] that maximum number of shoots was initiated when potato nodes are cultured in Muraghige and Skoog medium including growth regulators.

\section{Number of Nodes plant ${ }^{-1}$}

The average number of nodes plant ${ }^{-1}$ after six days of culturing indicated that the medium having TANG induced maximum (1.37) number of nodes plant ${ }^{-1}$ whereas the mediums supplemented with ARQ (1) and GA3 (1) induced similar number of nodes plant $^{-1}$ (Fig. 14). Statistical analysis shows that there was no significant difference between the natural growth substances and synthetic growth regulators.

The average number of nodes plant ${ }^{-1}$ after eight days of culturing indicated that the medium having TANG induced maximum (2.75) number of nodes Plant ${ }^{-1}$ while the medium supplemented with GA3 (2.5) and the medium containing ARQ (2.5) showed similar number of nodes plant ${ }^{-1}$ (Fig. 15). Statistical analysis shows that there was no significant difference between the natural growth substances and synthetic growth regulators. 
The average number of nodes plant $^{-1}$ after ten days of culturing indicated that the medium having TANG induced maximum (5.5) number of nodes plant $^{-1}$ and the medium supplemented with ARQ (3.5) second to it whereas the medium containing GA3 (3.25) was induced minimum number of shoots plant ${ }^{-1}$ (Fig. 16). Statistical analysis shows that there was no significant difference between the natural growth substances and synthetic growth regulators. The average number of nodes plant $^{-1}$ after thirteen days of culturing indicated that the medium having TANG induced maximum (8.5) number of nodes plant ${ }^{-1}$ and the medium supplemented with ARQ (8) was second to it whereas GA3 containing medium induced minimum (6.87) number of shoots plant ${ }^{-1}$ (Fig. 17). Statistical analysis shows that there was no significant difference between the natural growth substances and synthetic growth regulators. The average number of nodes plant ${ }^{-1}$ after fifteen days of culturing indicated that the medium supplemented with TANG induced maximum (12.87) number of nodes plant ${ }^{-}$ ${ }^{1}$ and the medium having ARQ (12.25) was second to it whereas the medium containing GA3 induced minimum (9) number of shoots plant ${ }^{-1}$ (Fig. 18). Statistical analysis shows that there was no significant difference between the natural growth substances and synthetic growth regulators. Our results also supported by the findings of Ali et al. [23] who reported that natural growth regulators showed maximum increase in shoot length compared to synthetic.

The shoot length was measured in centimeter $(\mathrm{cm})$. The data recorded after six days of culturing indicated that the medium containing ARQ induced maximum (1.05) shoots length plant $^{-1}$ and the medium supplemented with TANG (0.96) was second to it. Whereas: the medium having GA3 showed minimum (0.412) shoots length plant ${ }^{-1}$ (Fig. 19). Statistical analysis shows that there was significant difference between the natural growth substances and synthetic growth regulators.
The data recorded after eight days of culturing indicated that ARQ supplemented medium induced maximum (3.3) shoots length plant $^{-1}$ and the medium containing TANG (2.49) was second to it. Whereas the medium having GA3 showed minimum (1.65) shoots length plant ${ }^{-1}$ (Fig. 20). Statistical analysis showed that there was significant difference between the natural growth substances and synthetic growth regulators.

The data recorded after ten days of culturing indicated that the medium supplemented with ARQ induced maximum (3.46) shoots length plant ${ }^{-1}$ and the medium having TANG (2.6) was second to it. Whereas GA3 contained medium show minimum (2.3) shoots length plant $^{-1}$ (Fig. 21). Statistical analysis shows that there was no significant difference between the natural growth substances and synthetic growth regulators.

The data recorded after thirteen days of culturing indicated that ARQ supplemented medium (3.09) induced maximum shoots length plant $^{-1}$ and the medium containing TANG (2.92) was second to it. Whereas the medium having GA3 (2.52) show minimum shoots length plant ${ }^{-1}$ (Fig. 22). Statistical analysis shows that there was no significant difference between the natural growth substances and synthetic growth regulators. Similar results were also reported by Ali et al. [23].

The data recorded after fifteen days of culturing indicated that the medium supplemented with ARQ induced maximum (3.26) shoots length plant ${ }^{-1}$ and the medium having TANG (2.78) was second to it. Whereas the medium containing GA3 showed minimum (2.56) shoots length plant (Fig. 23). Statistical analysis shows that there was no significant difference between the natural growth substances and synthetic growth regulators. In green house better growth was observed in TANG followed by ARQ while minimum growth was observed in GA3 as shown in (Fig. $24 \& 25$ ). The above discussion indicates that there is no 
significant difference between natural growth regulators (TANG and ARQ) and synthetic growth regulator (GA3). However, throughout the experiment natural growth regulators (TANG and ARQ) was much more efficient in overall growth of potato than synthetic growth regulator (GA3). The study reveals that (i) Natural growth regulators are suitable substitute of synthetic growth regulator (ii) Natural growth regulators did not exhibit any disorder or adverse effect on the overall development and growth of potato plant (iii) bud proliferation completed in three days in both natural growth regulators and synthetic growth regulator (iv) the plants showed batter survival in natural growth regulators (v) The cost of production plant ${ }^{-}$ ${ }^{1}$ of potato for natural growth regulators is lower than the synthetic growth regulators. These results are in consistence with the findings of Ali et al. [23] who reported that synthetic growth regulator work much in the same way as natural growth regulators present in plants while Anonymous [24] stated that large amount of plant growth regulators are hazardous for environment and human health and should be replaced by less hazardous compounds having natural source.

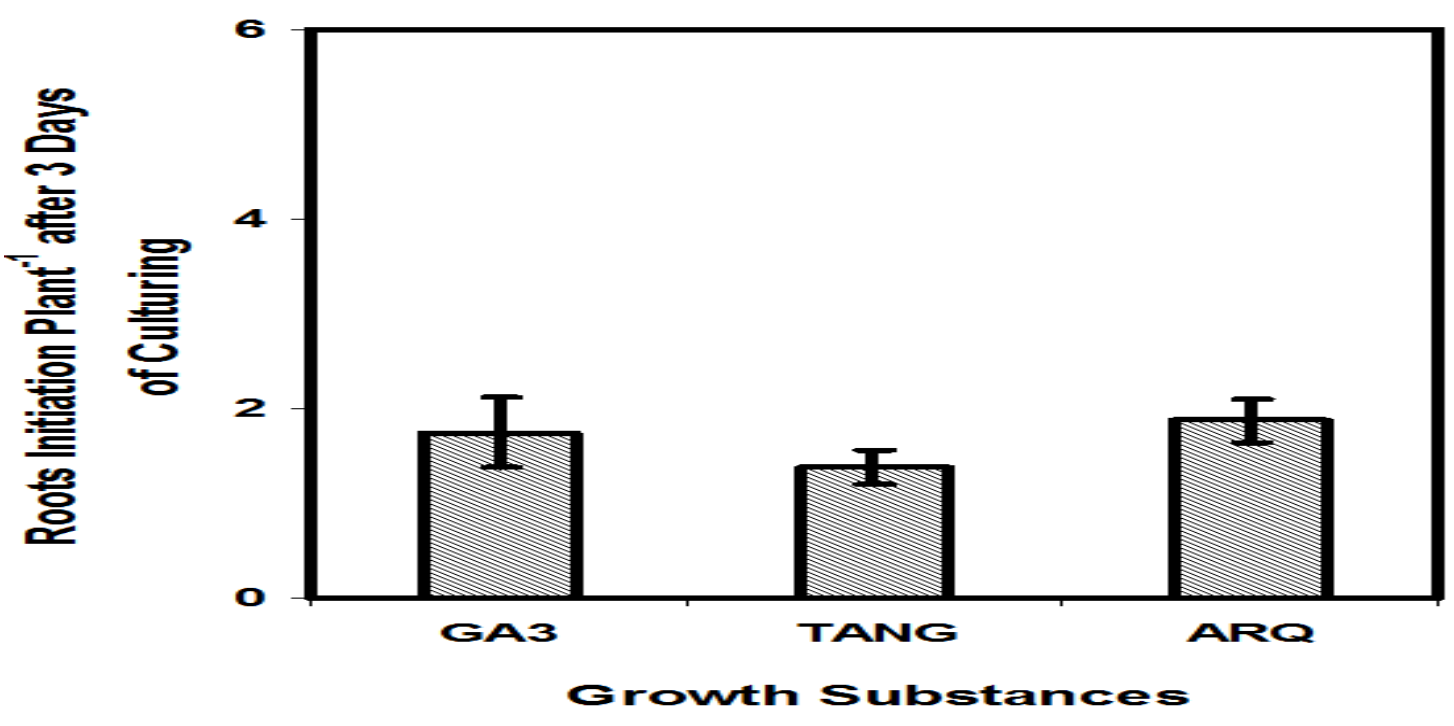

Figure. 1. Roots initiation after 3 days of culturing in M.S media supplement with different growth stimulators

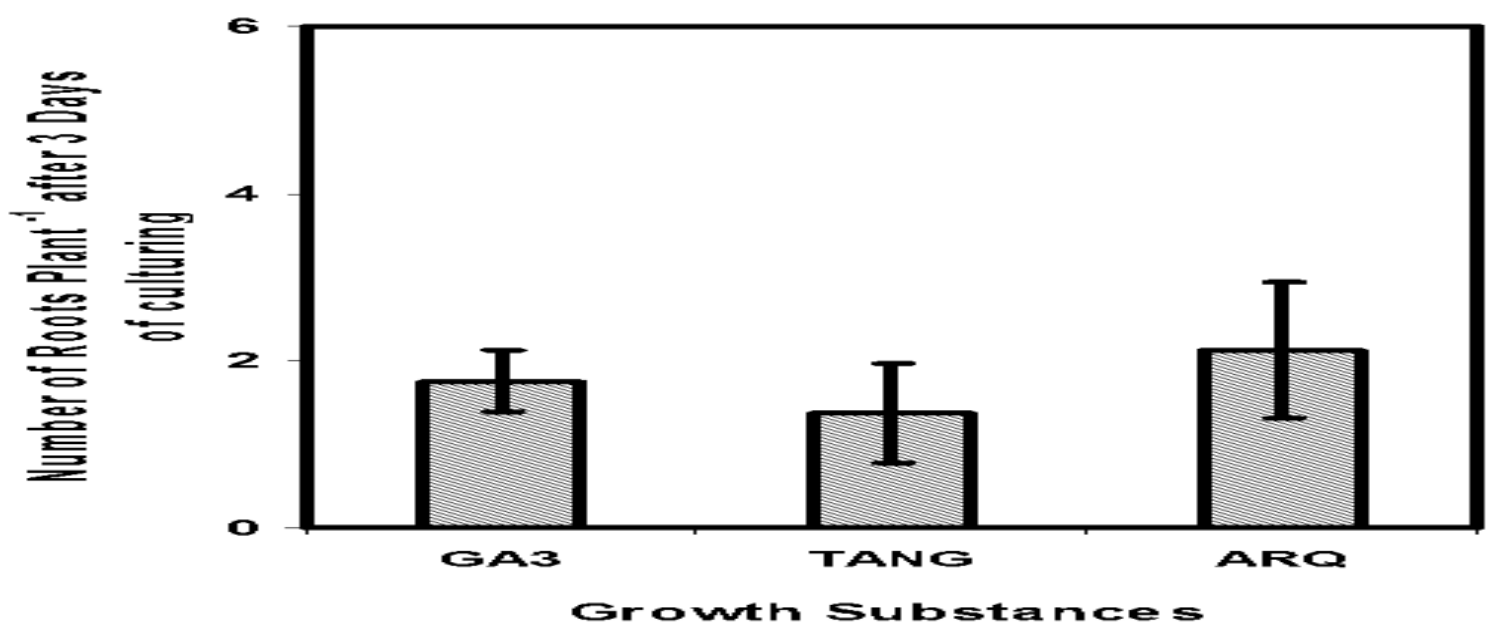

Figure 2. Number of roots plant ${ }^{-1}$ after 3 days of culturing in M.S media supplement with different growth stimulators 


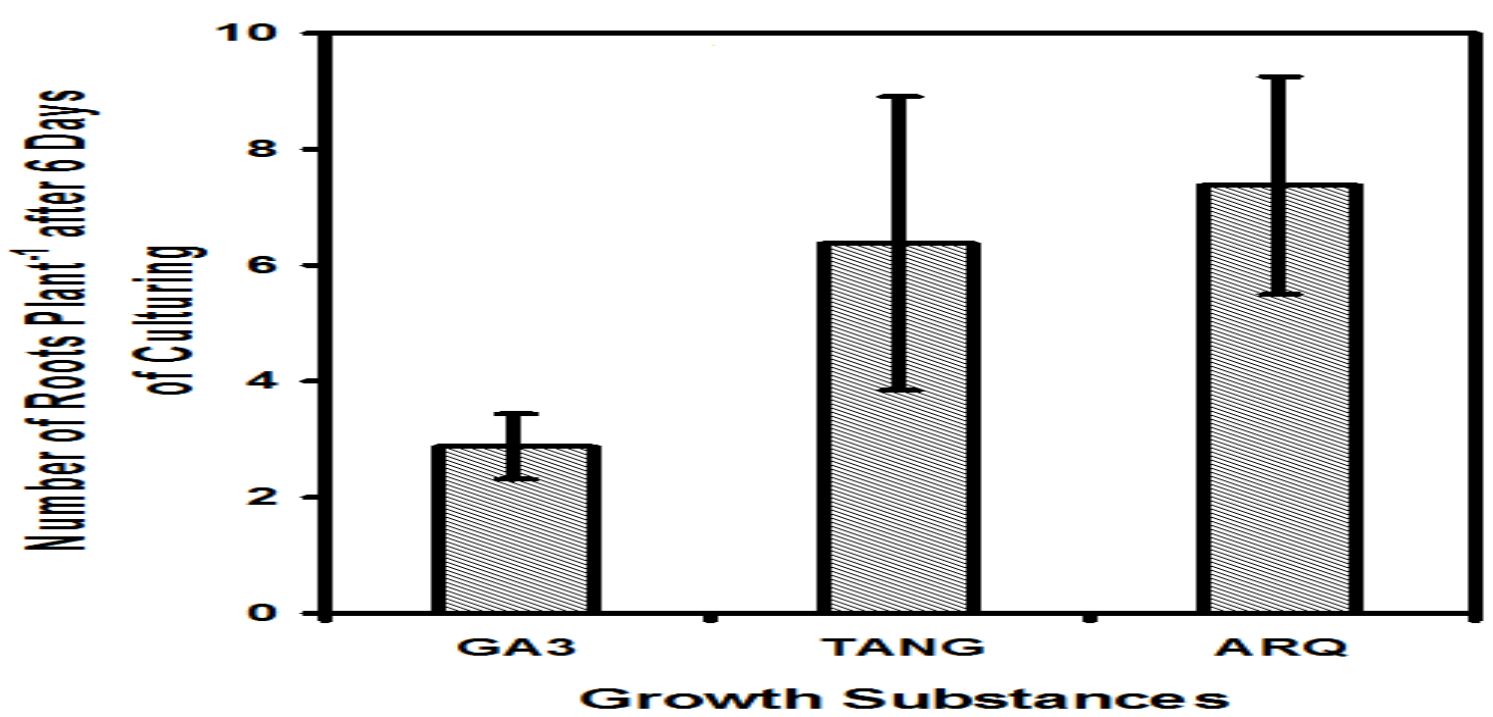

Figure 3. Number of roots plant ${ }^{-1}$ after 6 days of culturing in M.S media supplement with different growth stimulators

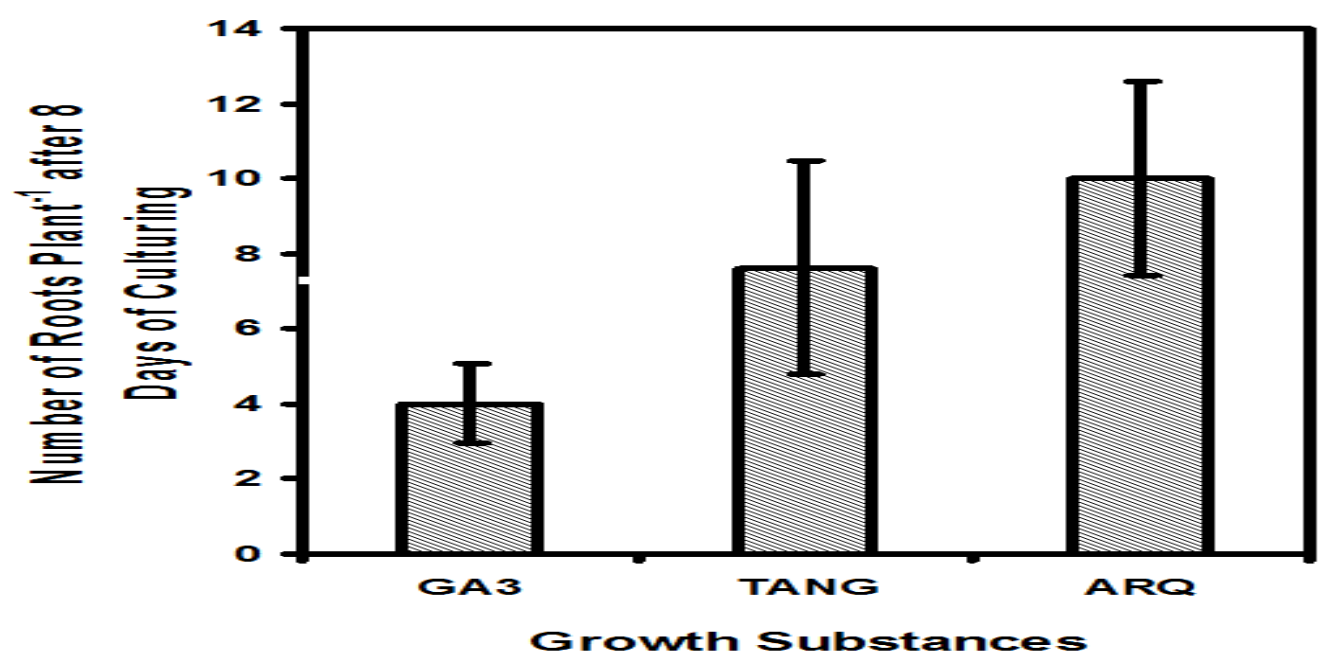

Figure 4. Number of roots plant ${ }^{-1}$ after 8 days of culturing in M.S media supplement with different growth stimulators

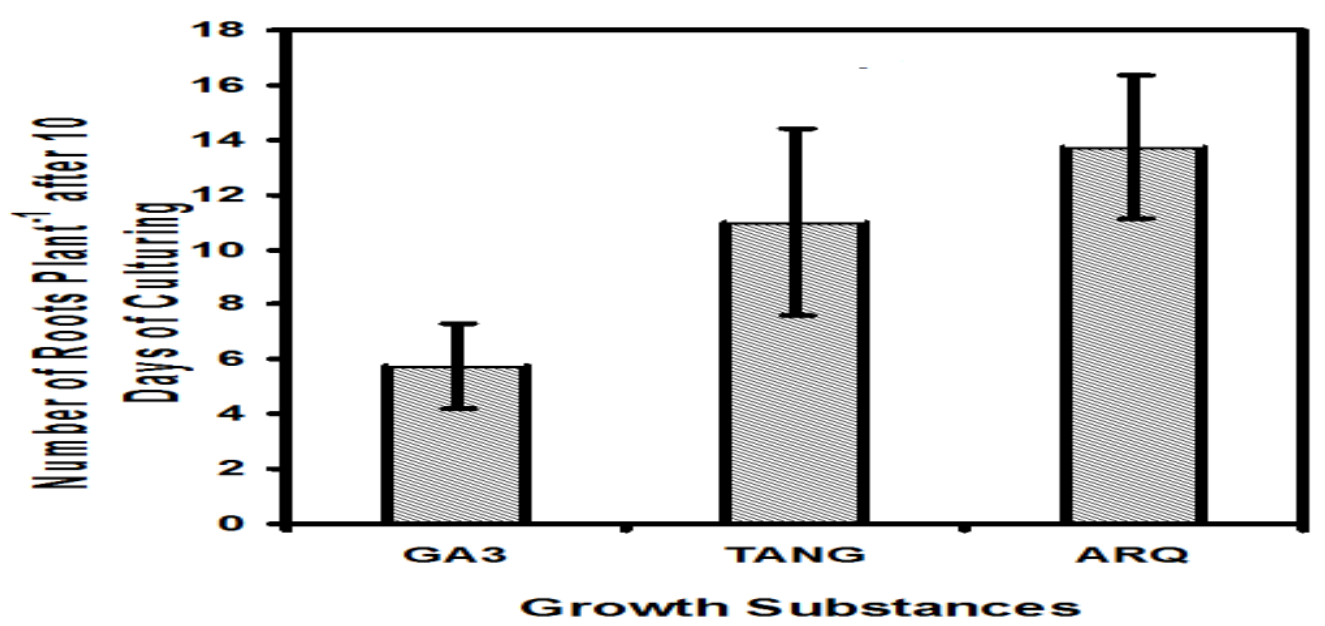

Figure 5. Number of roots plant ${ }^{-1}$ after 10 days of culturing in M.S media supplement with different growth stimulators 


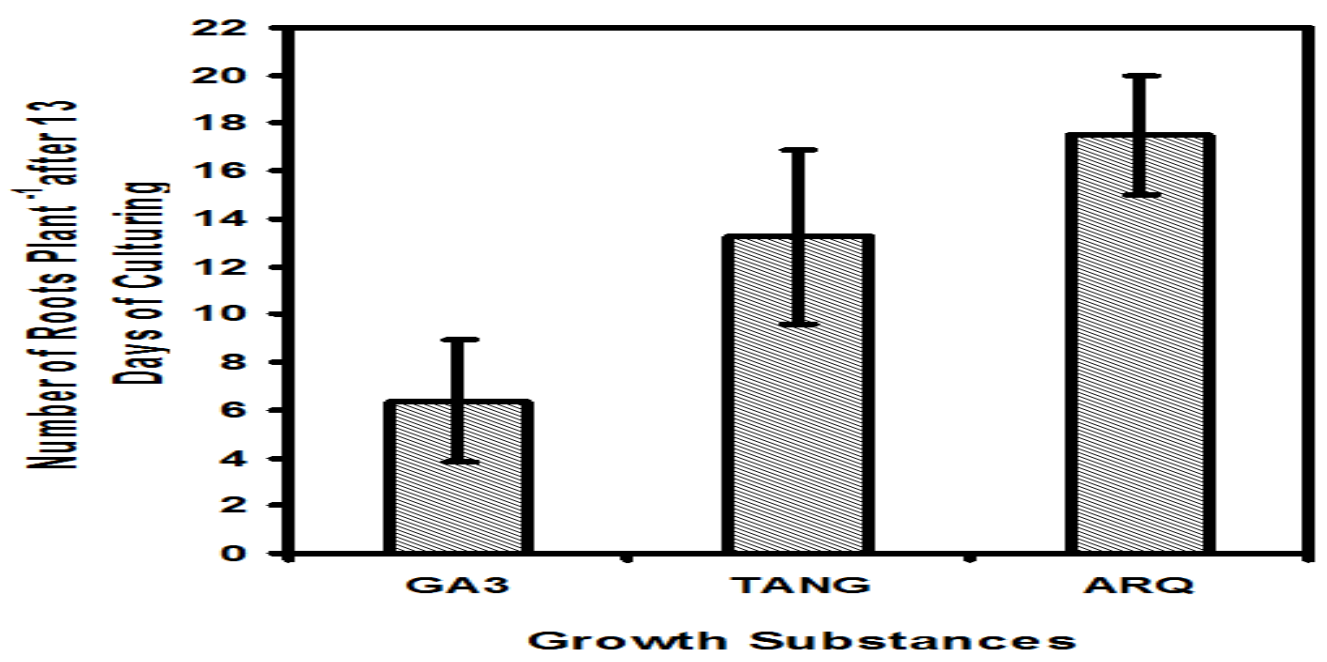

Figure 6. Number of roots plant ${ }^{-1}$ after 13 days of culturing in M.S media supplement with different growth stimulators

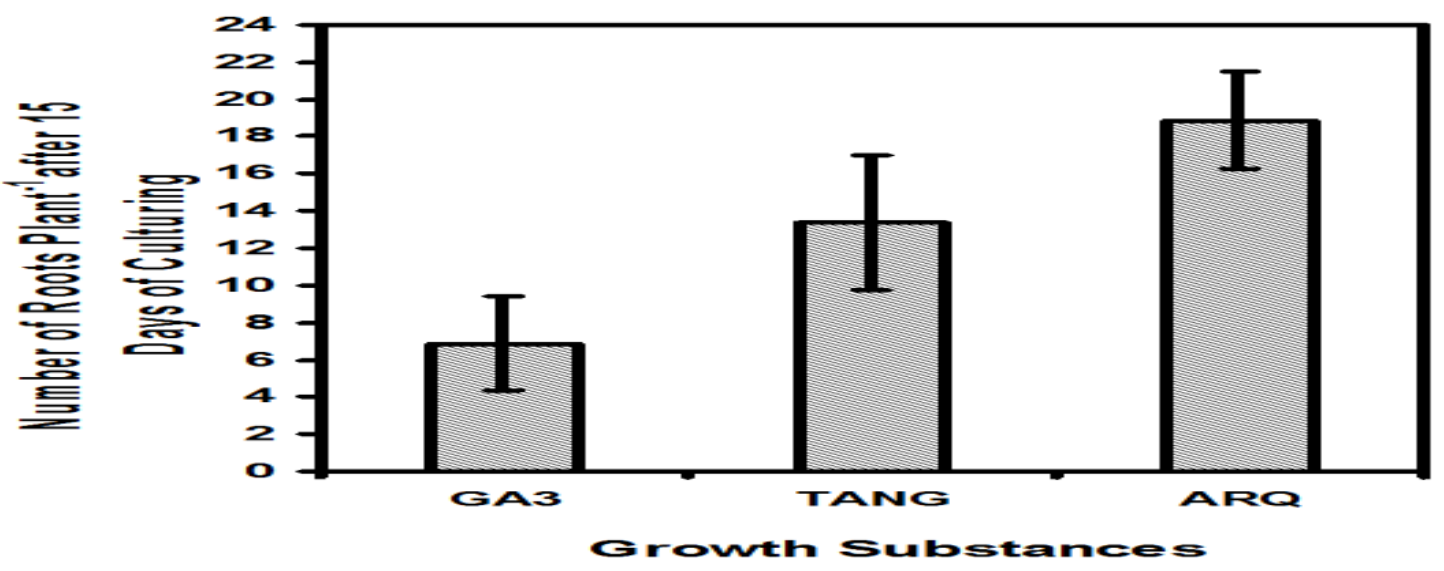

Figure 7. Number of roots plant ${ }^{-1}$ after 15 days of culturing in M.S media supplemented with different growth stimulators

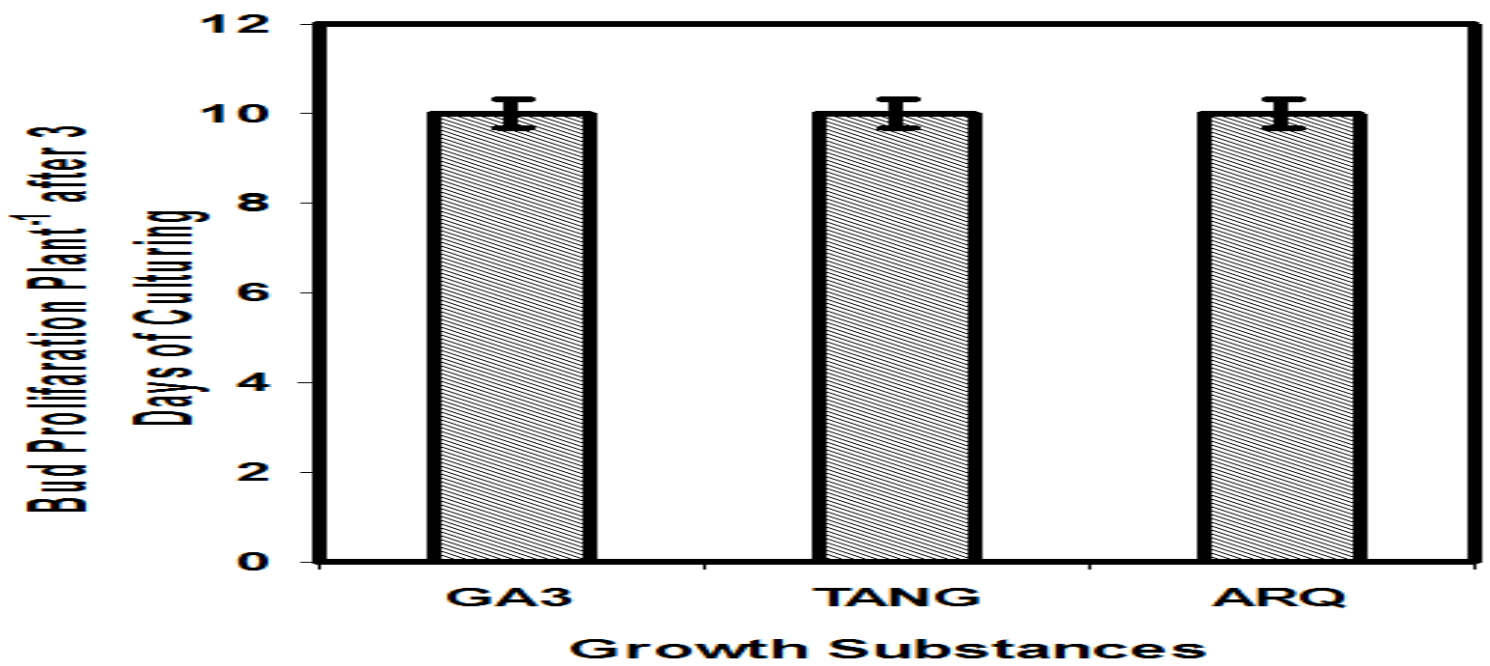

Figure 8. Bud proliferation plant ${ }^{-1}$ after 3 days of culturing on M.S media supplemented with different growth stimulators 


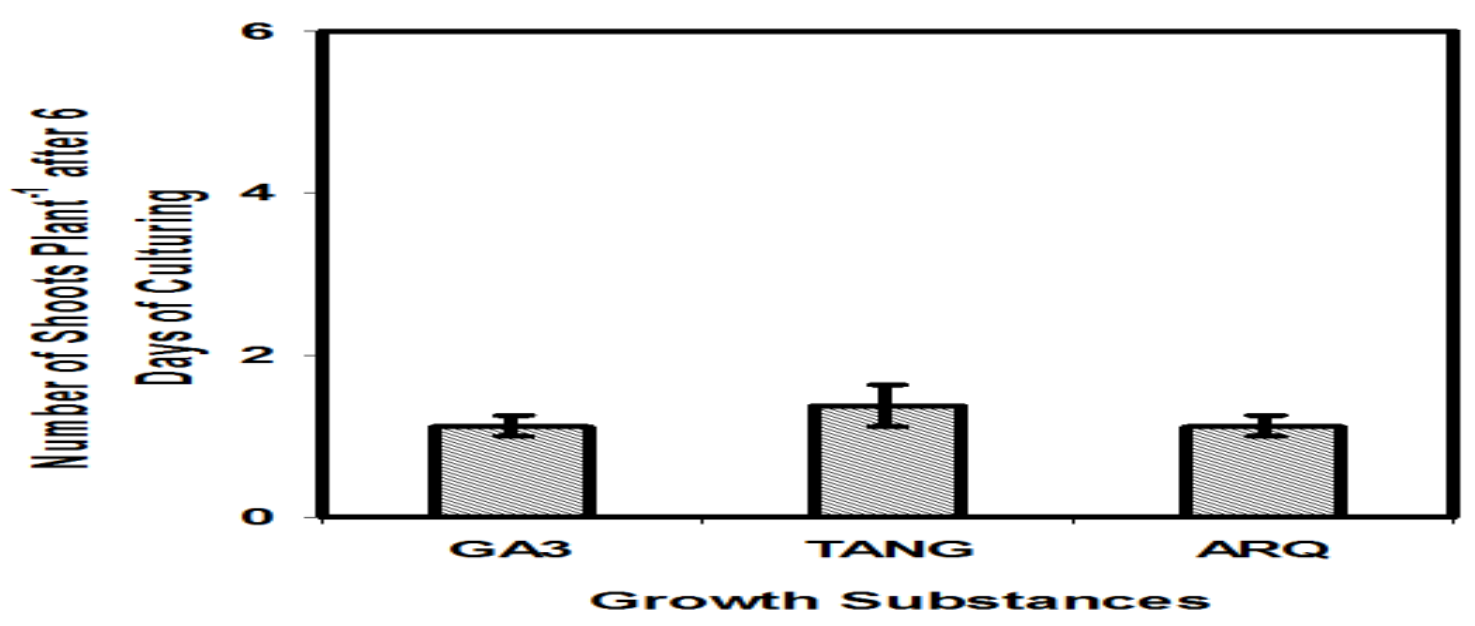

Figure 9. Number of shoots plant ${ }^{-1}$ after 6 days of culturing on M.S media supplemented with different growth stimulators

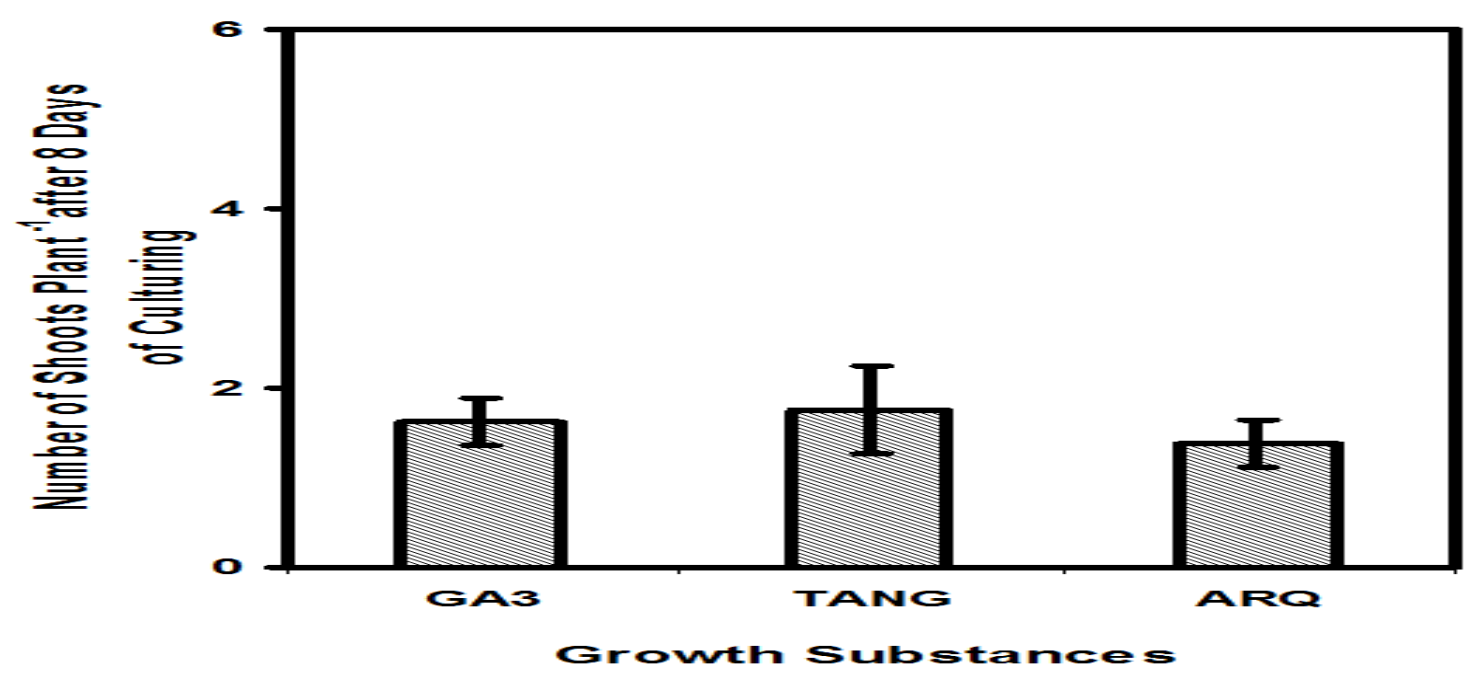

Figure 10. Number of shoots plant ${ }^{-1}$ after 8 days of culturing on M.S media supplemented with different growth stimulators

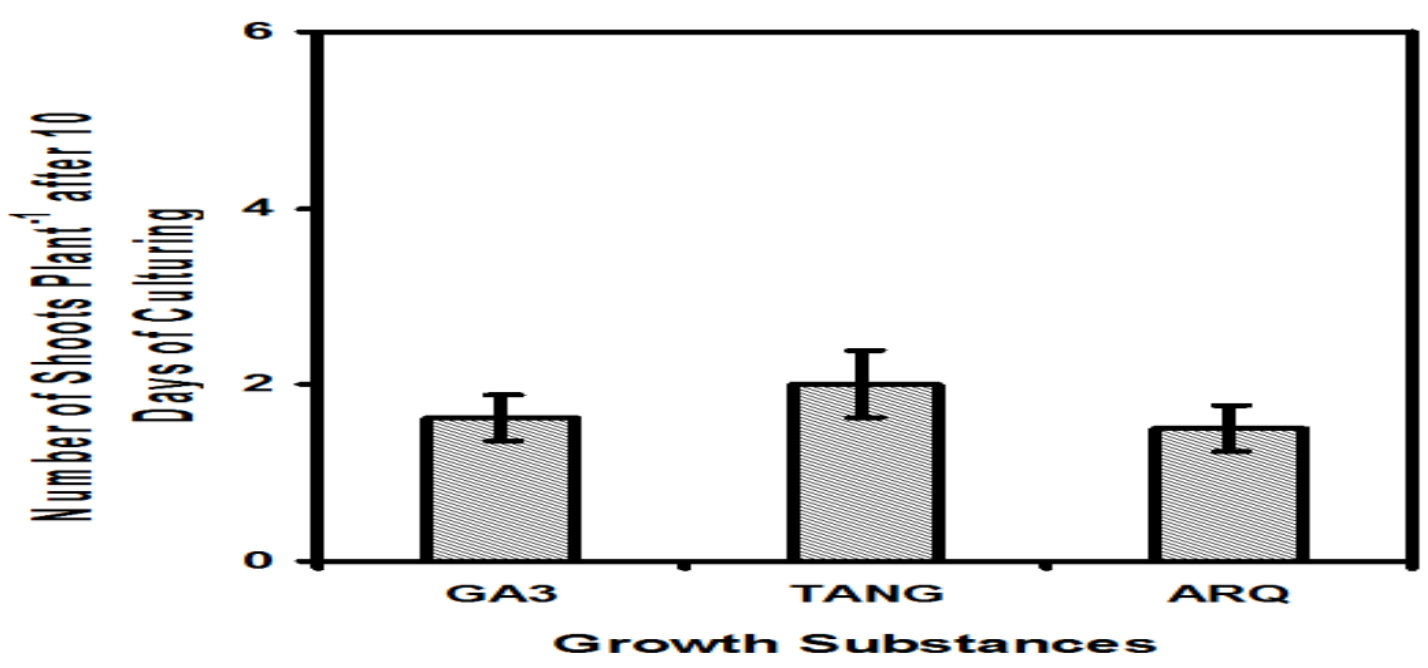

Figure 11. Number of shoots plant ${ }^{-1}$ after 10 days of culturing on M.S media supplemented with different growth stimulators 


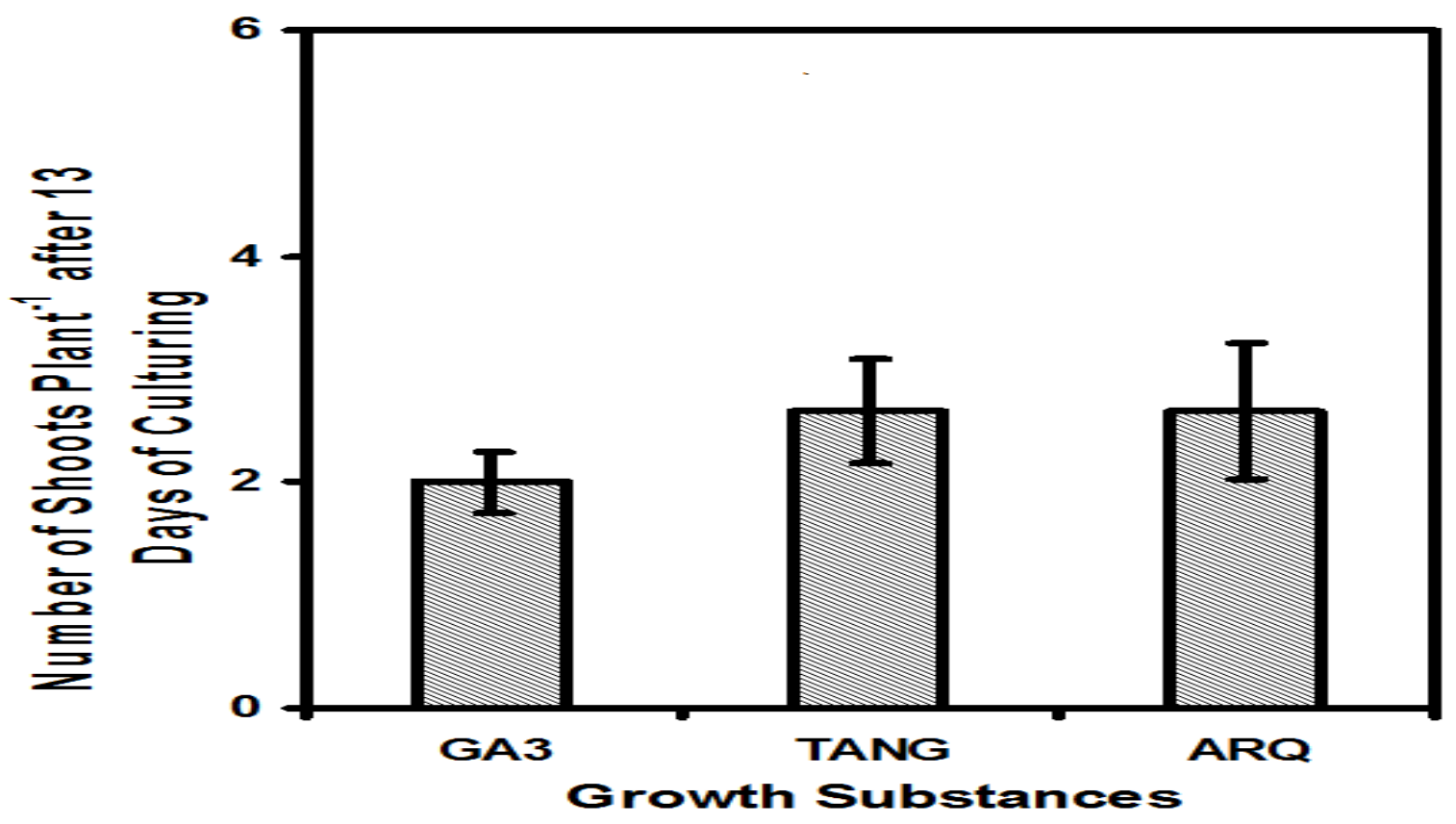

Figure 12. Number of shoots plant ${ }^{-1}$ after 13 days of culturing on M.S media supplemented with different growth stimulators

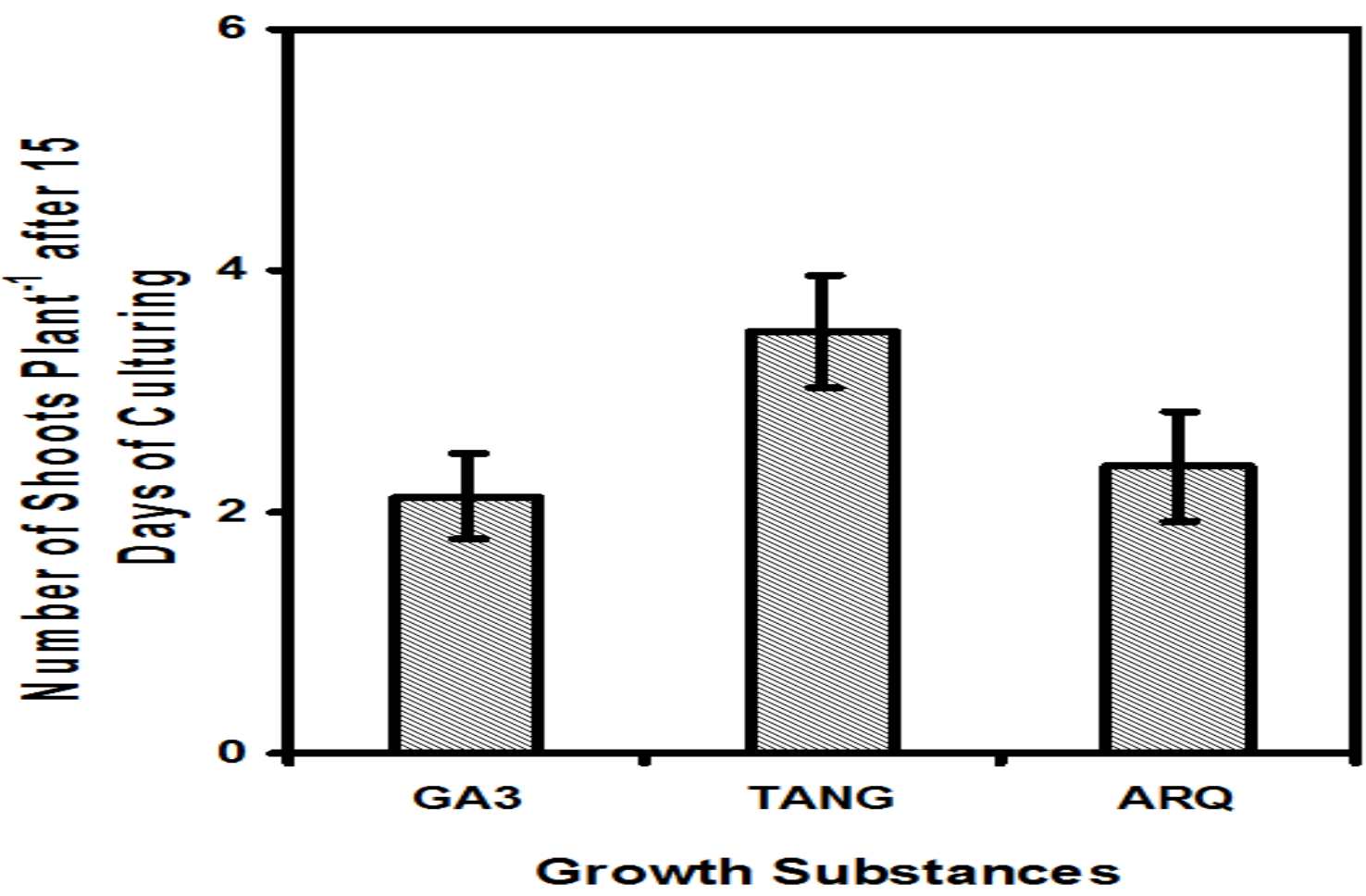

Figure 13. Number of shoots plant ${ }^{-1}$ after 15 days of culturing on M.S media supplemented with different growth stimulators 


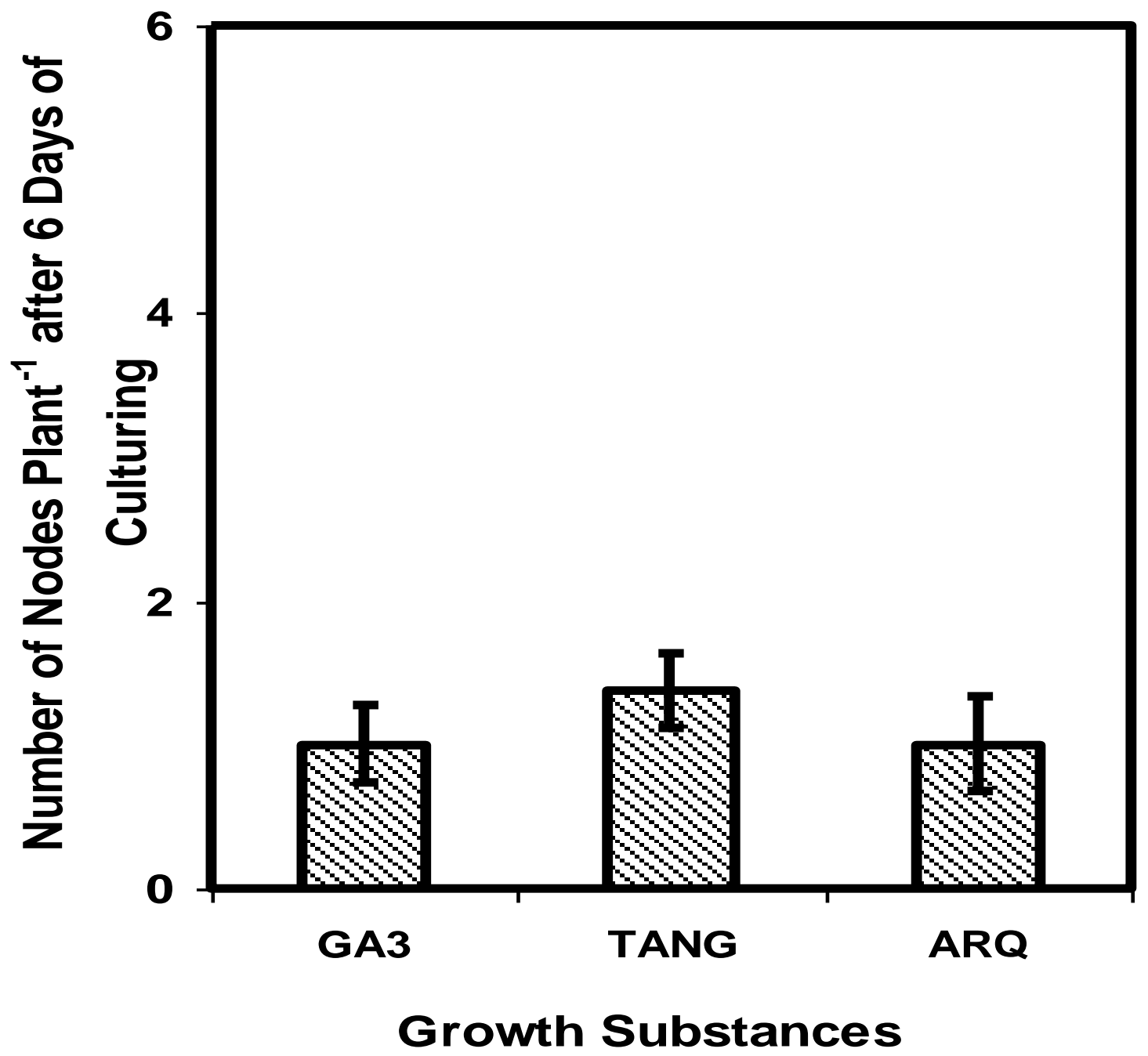

Figure 14. Number of nodes plant ${ }^{-1}$ after 6 days of culturing on M.S media supplemented with different growth stimulators

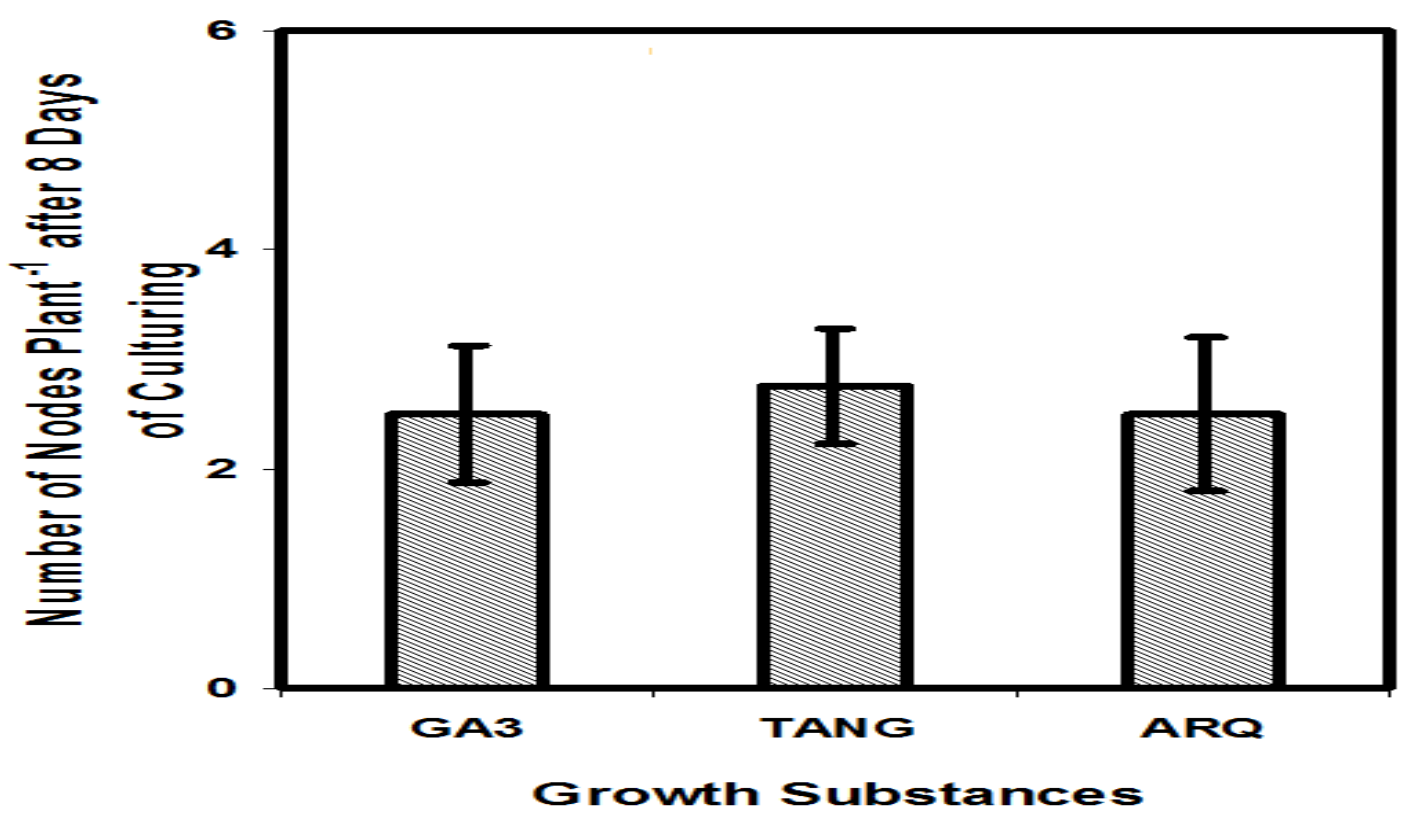

Figure 15. Number of nodes plant ${ }^{-1}$ after 8 days of culturing on M.S media supplemented with different growth stimulators 


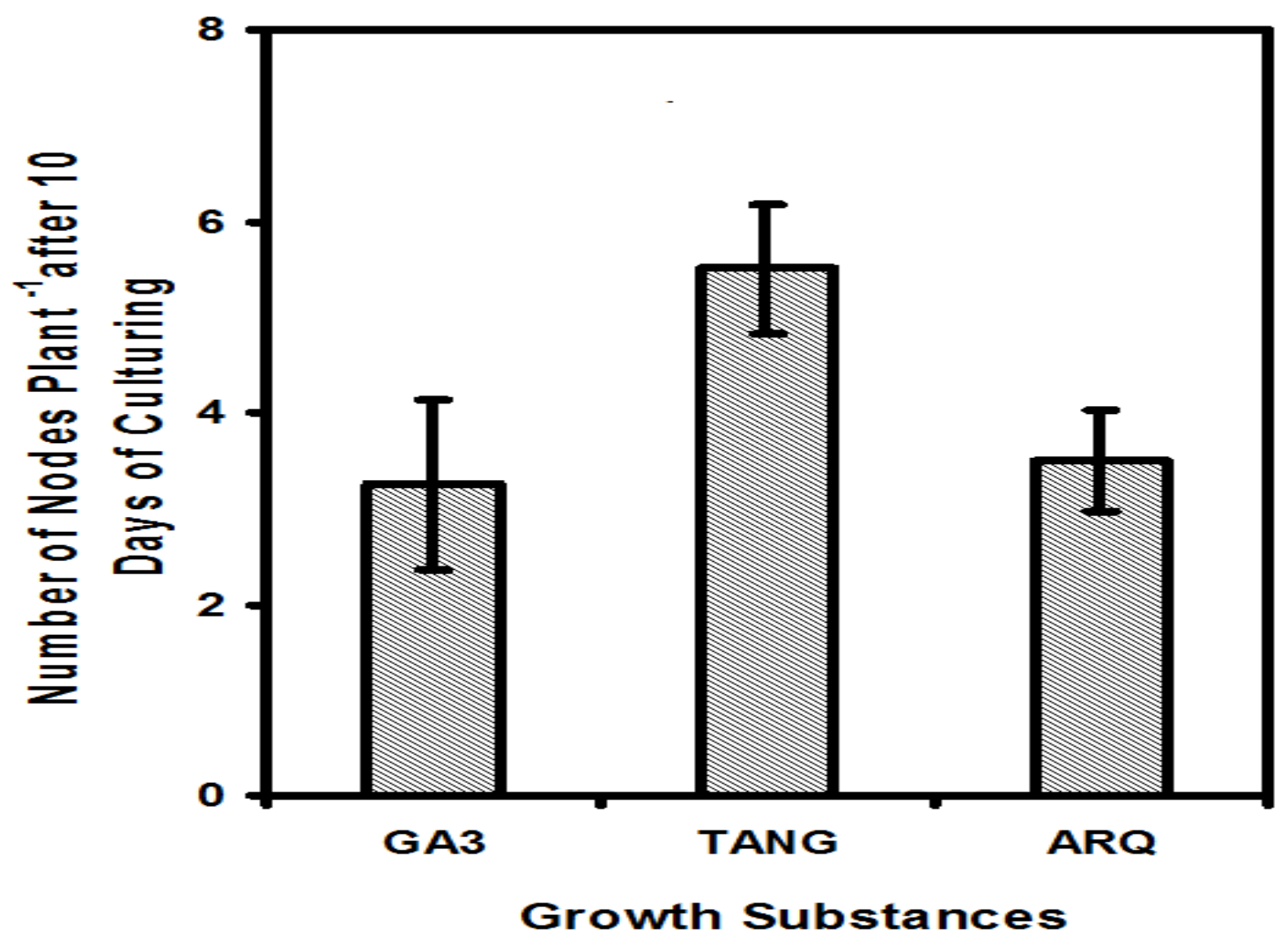

Figure 16. Number of nodes plant ${ }^{-1}$ after 10 days of culturing on M.S media supplemented with different growth stimulators

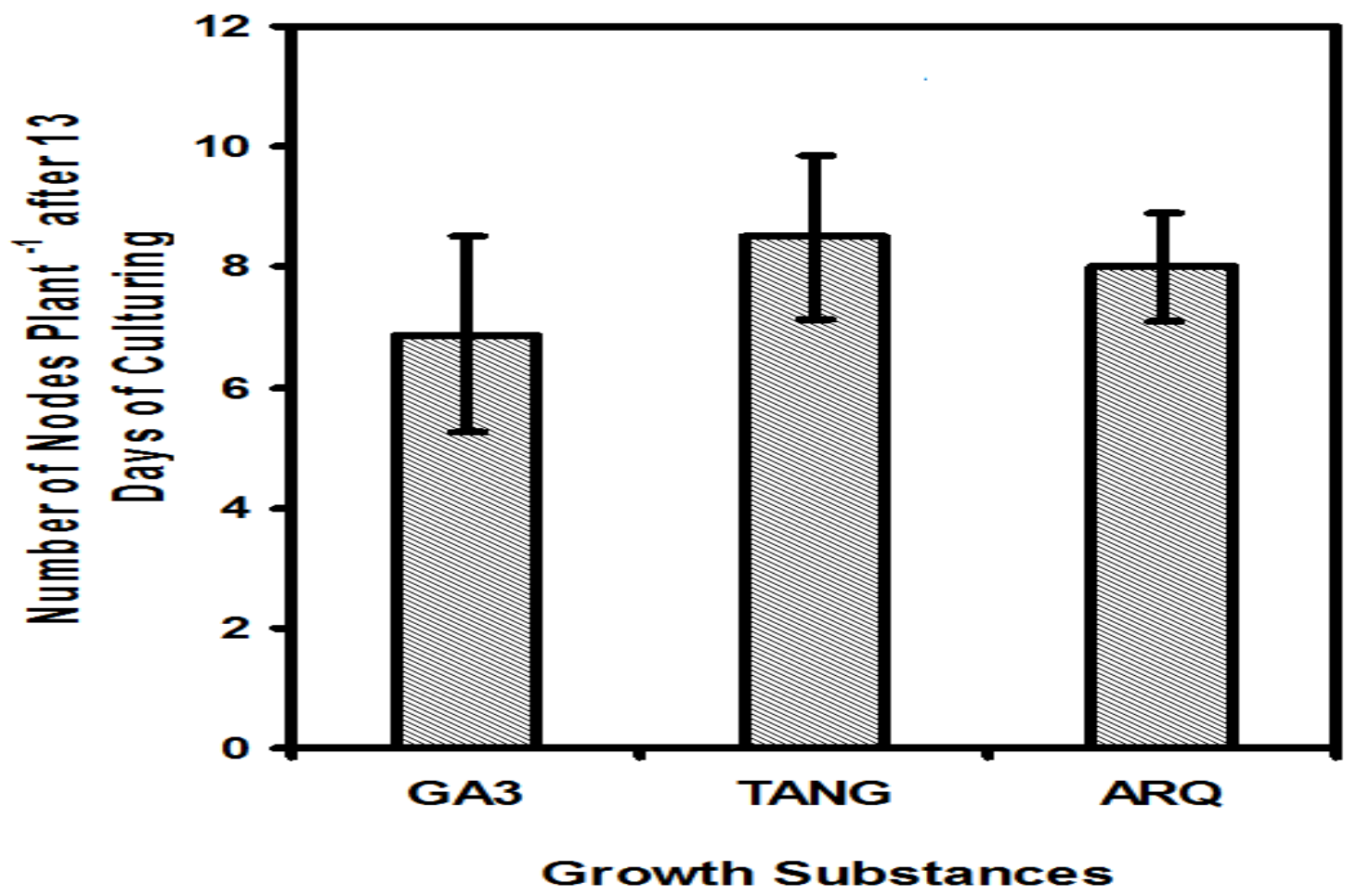

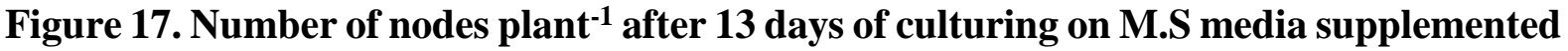
with different growth stimulators 


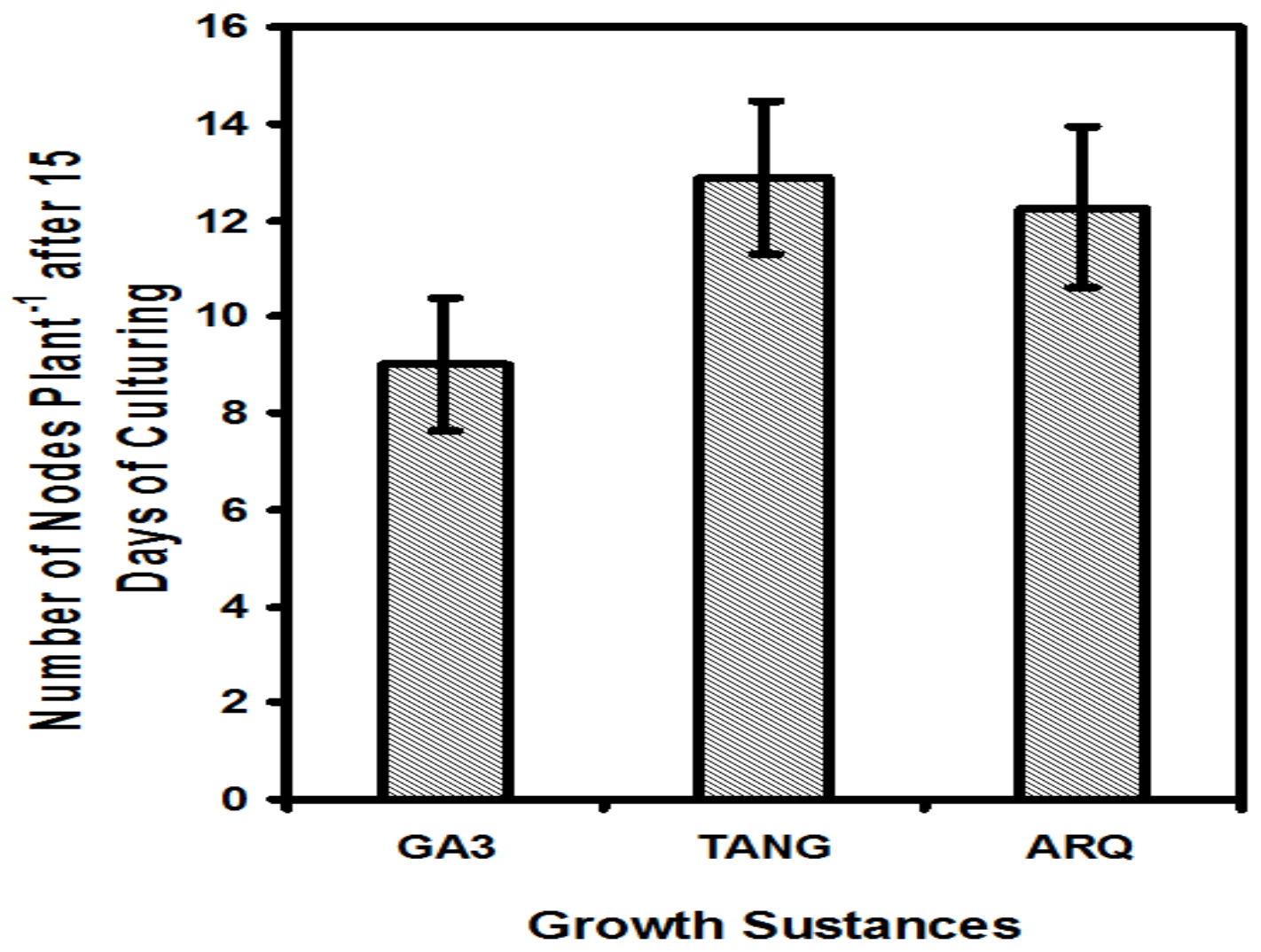

Figure 18. Number of nodes plant ${ }^{-1}$ after 15 days of culturing on M.S media supplemented with different growth stimulators

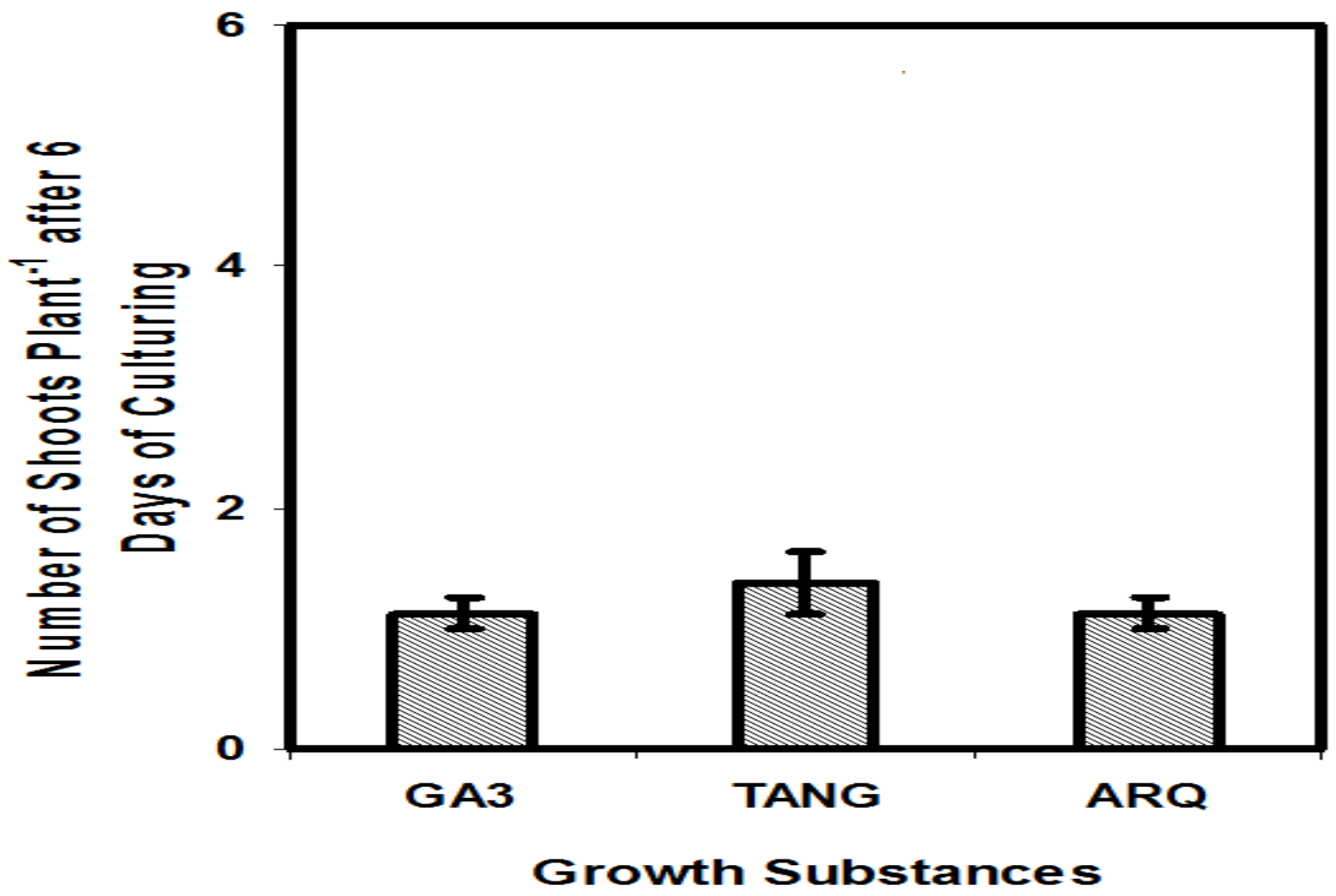

Figure 19. Shoot length plant $^{-1}$ after 6 days of culturing on M.S media supplemented with different growth stimulators 


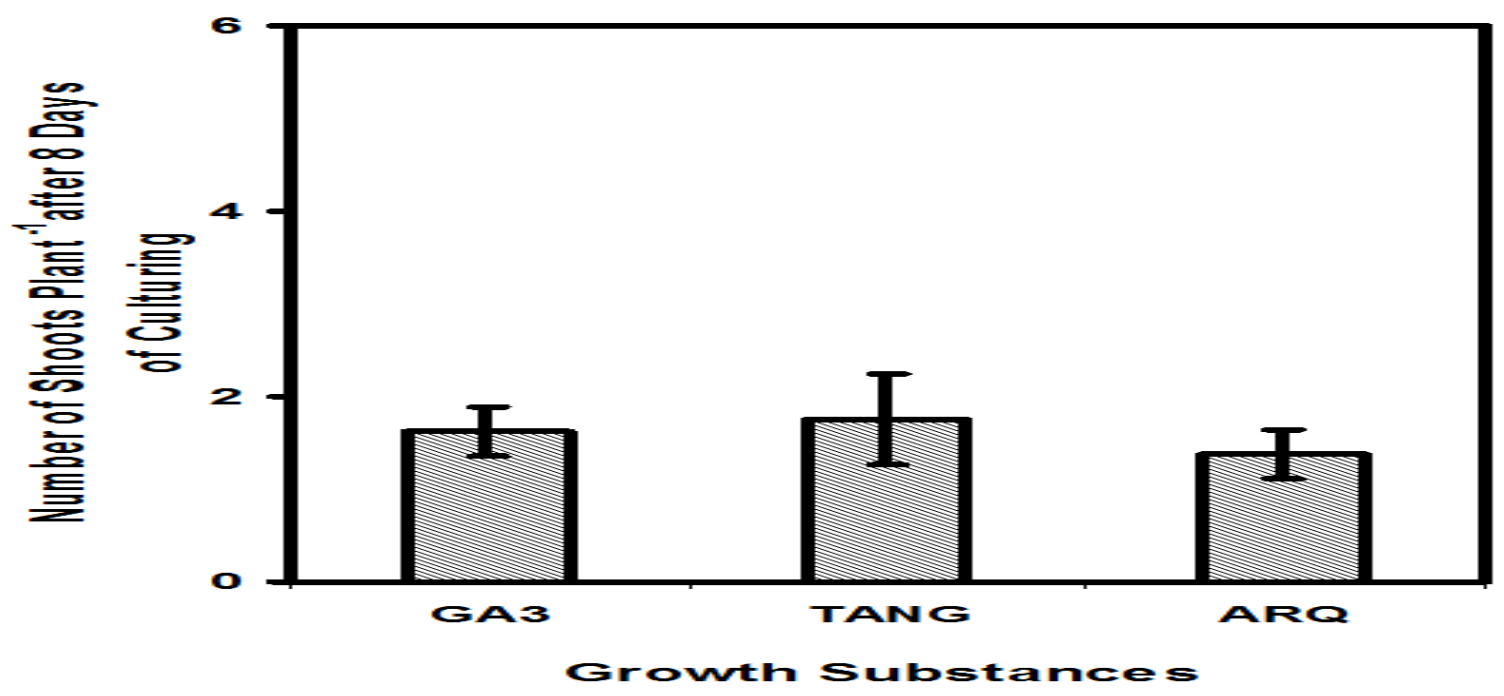

Figure 20. Shoot length plant ${ }^{-1}$ after 8 days of culturing on M.S media supplemented with different growth stimulators

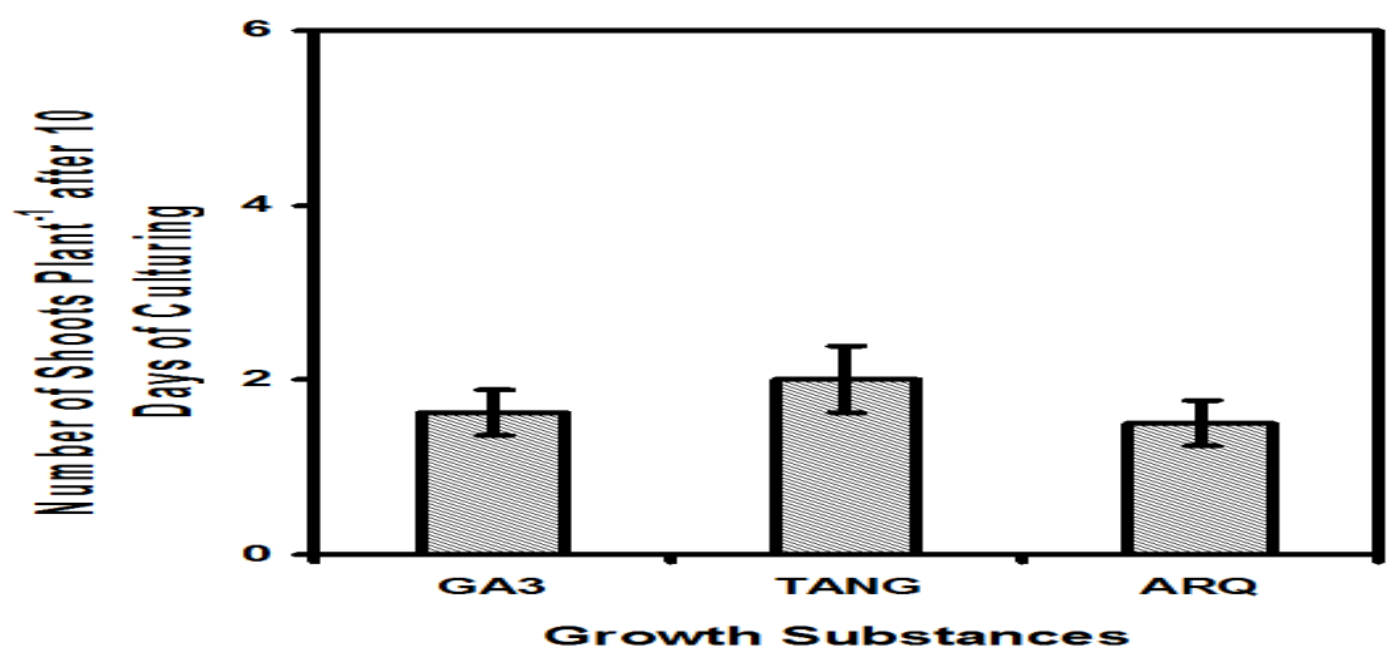

Figure 21. Shoot length plant ${ }^{-1}$ after 10 days of culturing on M.S media supplemented with different growth stimulators

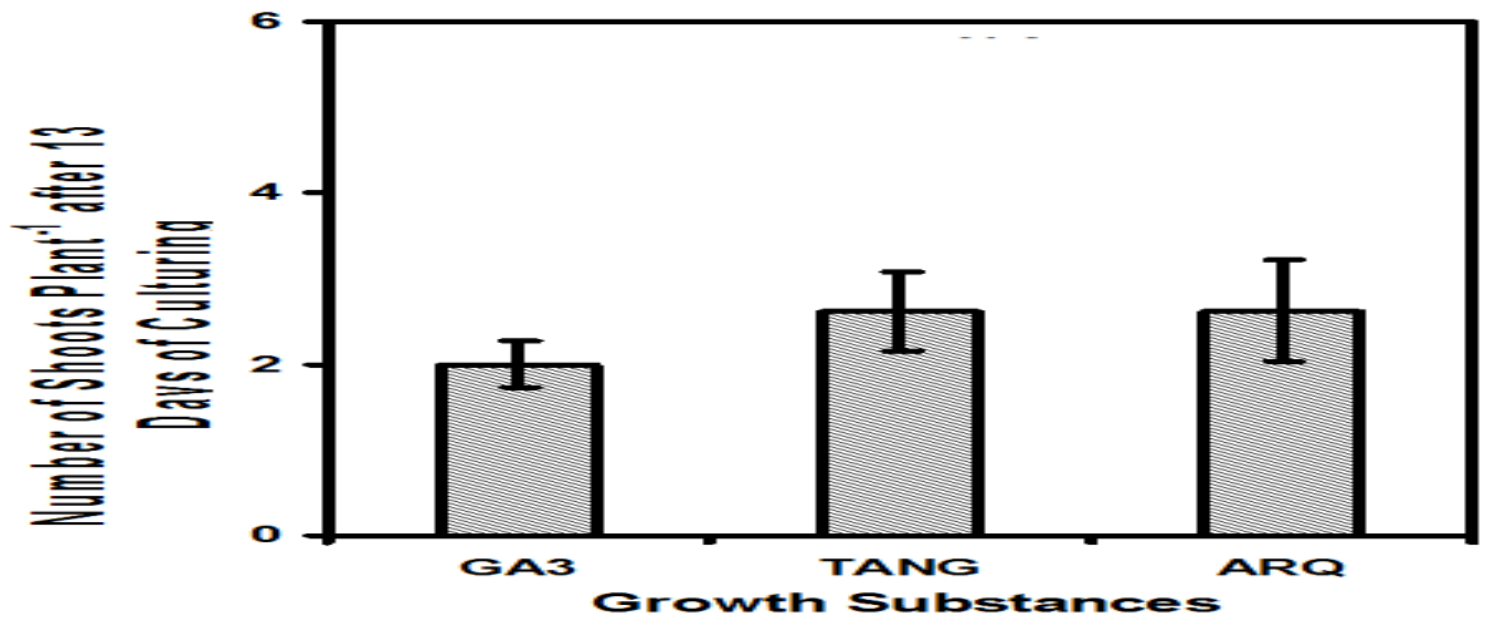

Figure 22. Shoot length plant $^{-1}$ after 13 days of culturing on M.S media supplemented with different growth stimulators 


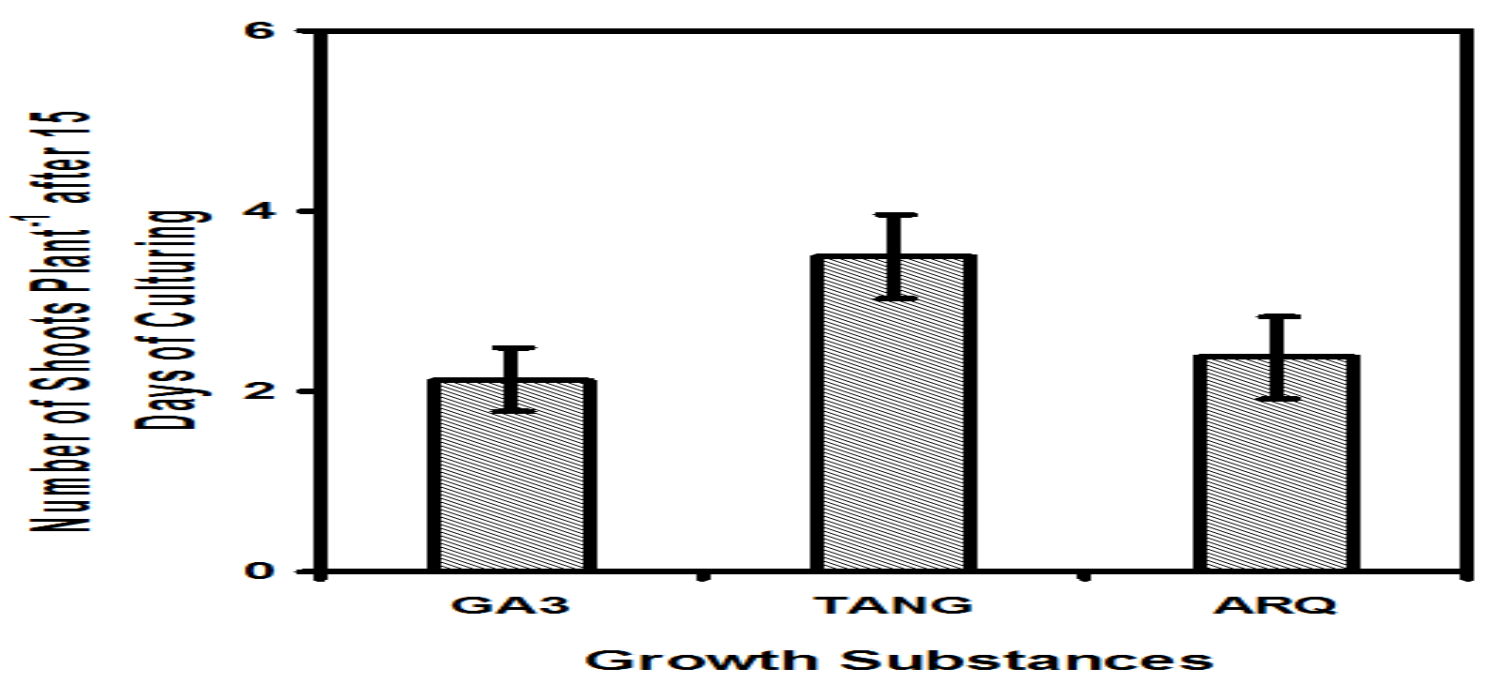

Figure 23. Shoot length plant ${ }^{-1}$ after 15 days of culturing on M.S media supplemented with different growth stimulators

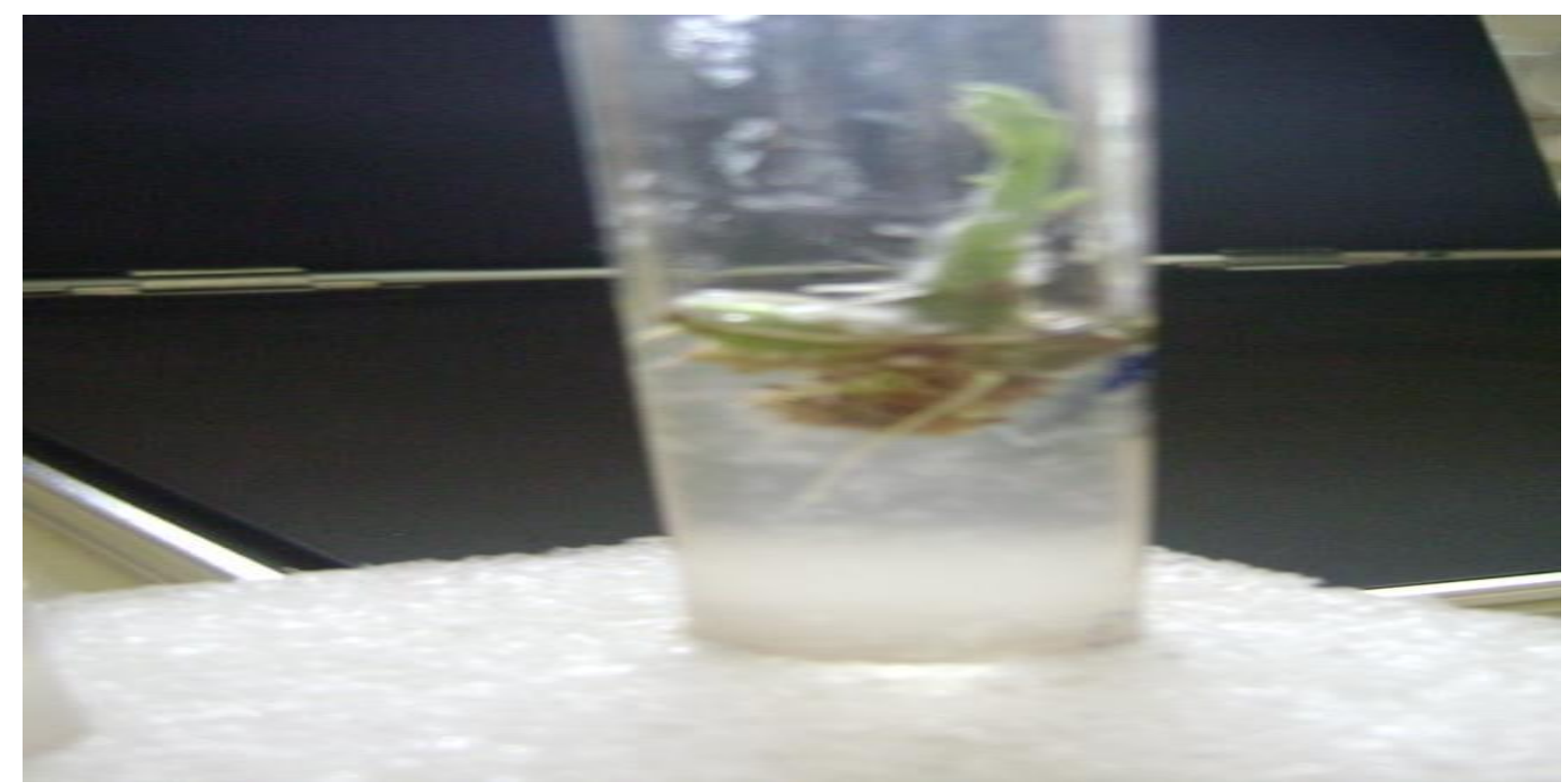

Figure 24. Roots initiation in the medium supplemented with ARQ after 3 days

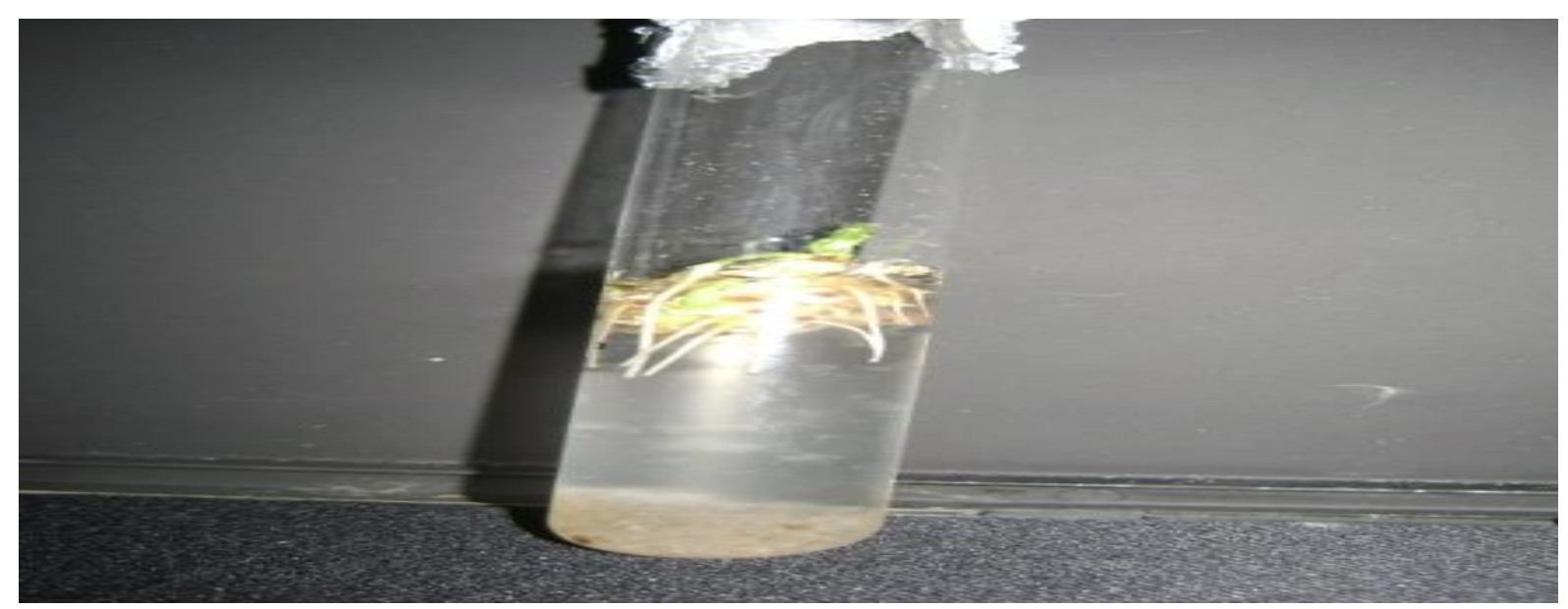

Figure 25. Roots initiation in the medium supplemented with TANG after 3 days 


\section{Conclusions}

All these evidences strongly suggest that natural growth regulators / substances are better to be used instead of synthetic growth regulator because natural growth regulators are the cheaper substitute of synthetic growth regulator. There is every possibility of natural growth exacts becoming a universal growth regulators.

\section{Authors' contributions}

Conceived and designed the experiments: ZU Nisa, SH Shah \& AH Shah. Performed the experiments: ZU Nisa, Analyzed the data: ZU Nisa, SH Shah \& AH Shah, Contributed materials/ analysis/ tools: G Farooq, SH Shah, AH Shah \& MA Saleem, Wrote the paper: ZU Nisa \& MA Sajad.

\section{Acknowledgment}

The research was conducted in the Institute of Biotechnology and Genetic Engineering (IBGE) Khyber Pakhtunkhwa Agricultural University, Peshawar. Potato plants were used for experimentation.

\section{References}

1. Haris PM (1992). The potato crop. Chapman and Hall, USA, New York.

2. Rahman MM, Amin MN \& Ahmad S (2003). Rapid clonal propagation of 'Native Olive' (Elaercarpus srobustus Roxb.) using tissue culture technique. J Biol Sci 3(12): 1107-1113.

3. GOP (2011). Economic Survey of Pakistan, 2010-11. Government of Pakistan, Finacae division Economic advisor's wing, Islamabad, pp 21-22.

4. Withers LA, Weheelans SK \& Williams JT (1990). In vitro conservation of crop germplasm and the IBPGR databases. 45(3): 9-22.

5. Halterman DA, Charkosawki \& Verchot J (2012). Potato viruses and seed certification USA. Pest technologies Global Science Book.

6. Robert, Y, Woodford JAT \& Ducray Bourdin DG (2000). Some epidemiological approaches to the control of aphid borne virus diseases in seed potato crops in Northern Europe, Virus Res 15: 33-47.
7. Reddy GM (2006). Clonal propagation of bamboo (Dendrocalamus strictus)/ Curr Sci 91(11):1462-1464.

8. Konstas J \& Kintzios S (2003). Developing a seed workshop 4-5 Sep. 1993, Quetta. Pak-Swiss Potato Dev. Proj. PARC, Islamabad.

9. Street HE (1977). Plant Tissue and Cell Culture. Blackwell Sci Publ Water J Chem Soc 528-537.

10. Nisa ZU, Jan S \& Shah SH (2016). Standardization of Protocol for the Surface Sterilization and Callus Induction of Saussurea lappa: An Endangered Medicinal plant. IJB 9(1): 324-330.

11. Nisa ZU, Jan S, Sajad MA, Shah SH, Farooq G \& Ali H (2018). Micropropagation through apical shoot explants and morphogenic potential of different explants of Saussurea lappa: An endangered medicinal plant. Pure Appl Biol 8(1): 585-592.

12. Nisa ZU, Shah SH, Farooq G, Sajad MA, \& Khan MAS (2019). Identification, quantification and comparative assessment of costunolide in wild, cultivated and in vitro callus culture of Saussurea lappa by HPLC technique. Pure Appl Biol 8 (1): 910919.

13. Nisa ZU, Shah SH, Farooq G, Sajad MA \& Khan MAS (2019). Distribution and comparison of major selected elements in root of wild plant, root derived calli and various anatomical parts of cultivated Saussurea lappa: An endangered medicinal plant. Pure Appl Biol 8(2): 1478-1486.

14. Nisa ZU, Jan S, Shah SH, Farooq G, Sajad MA \& Khan MAS (2019). Assessment of the callus induction, relative growth rate, proline and sugar in Saussrea lappa CB Clarke. Pure Appl Biol 8(1): 838-846.

15. Martinez J, Steenbergen S \& Vimr E (1995). Derived structure of the putative sialic acid transporter from Escherichia coli predicts a novel sugar permease domain. J Bacteriol 177: 
6005-6010.

16. Terlizzi DE, Ferrier MD, Armbrester EA \& Anauf KA (2002). Inhibition of dinoflagellate growth by exeracts of barely straw (Hordeum vugare). $J$ Applied Phycol 14(4): 275-280.

17. Raguraman S \& Singh (1998). Behavioral and Physiological effects of neem (Azadiracta indica) seed kernel extracts on larval parasitoid, Bracon herbetor. J Chem Ecol 24(7): 12411250 .

18. Ahmad, N, Muhammad A, Khan SA, Nasir AK, Rana B, Ahmad FS \& Rehman A (2011). Epidemiological Studies and Management of Potato Germplasam against pvx and pvy pak. J Phytopathol 23(2): 159-165.

19. George EF (2008). Plant Propagation by Tissue Culture 3rd Edition, 175204.

20. Mohammadi J \& Sivritepe N (2007). In vitro clonal propagation of Cucumis sativus L. by shoot tip culture. J Biol Sci 7(4): 653-657.

21. Shah AH, Shah SH, Swati ZA \& Hussain Z (2003). Cost Effective Micropropagation Technology for Potato. Pak J Biol Sci 6(4): 336-340.

22. Wang JJ, He, Wan Y, and Duan J (1990). Improvement in inducing in vitro tubers of potato (Solanum spp.). CIP, Region VIII: Chinese Academy of Agricultural Sciences (CAAS). Potato and Sweet Potato Res in China 63(1): 135-140.

23. Ali B, Hayat S, Fariduddin Q \& Ahmad A (2008) 24-Epi-brassinolide protects against the stress generated by salinity and nickel in Brassica juncea. Chemosphere 72: 1387-1392.

24. Anonymous (2003). Fruit, vegetables, and condiments statistics of Pakistan. Ministry of Food Agriculture and Livestock (Econ. Wing), Islambad, pp 11-12. 